\title{
Cellular perception of growth rate and the mechanistic origin of bacterial growth laws
}

\author{
Chenhao $\mathrm{Wu}^{1, \dagger}$, Rohan Balakrishnan ${ }^{1, \dagger}$, Matteo Mori ${ }^{1}$, Gabriel Manzanarez ${ }^{2}$, \\ Zhongge Zhang ${ }^{2}$, Terence Hwa ${ }^{1,2, *}$ \\ ${ }^{1}$ Department of Physics \& ${ }^{2}$ Division of Biological Sciences, \\ University of California at San Diego, La Jolla, California 92093, USA \\ $\dagger$ These authors contributed equally to this work. \\ *correspondence should be addressed to: Terence Hwa (hwa@ucsd.edu)
}

\begin{abstract}
Cells organize many of their activities in accordance to how fast they $\operatorname{grow}^{1-4}$. Yet it is not clear how they perceive their rate of growth, which involves thousands of reactions. Through quantitative studies of $E$. coli under exponential growth and during growth transitions, here we show that the alarmone ppGpp senses the rate of translational elongation by ribosomes, and together with its roles in controlling ribosome biogenesis and activity ${ }^{5-9}$, closes a key regulatory circuit that enables the cell to perceive the rate of its own growth for a broad class of growth-limiting conditions. This perception provides the molecular basis for the emergence of simple relations among the cellular ribosome content, translational elongation rate, and the growth rate, as manifested by bacterial growth laws ${ }^{2,10-13}$. The findings here provide a rare view of how cells manage to collapse the complex, high-dimensional dynamics of the underlying molecular processes to perceive and regulate emergent cellular behaviors, an example of dimension reduction performed by the cells themselves.
\end{abstract}

Guanosine tetraphosphate (ppGpp) $)^{14}$ is a key signaling molecule involved in bacterial response to environmental changes and has been extensively studied ${ }^{7-9,15-17}$, both for mechanisms contributing to its production and for its downstream effects on hundreds of genes ${ }^{18-21}$, including the synthesis $^{6,22}$ and activity ${ }^{7,9}$ of the translation machinery. Without a doubt, ppGpp-signaling plays a crucial role in responding to the cellular growth rate. Yet, despite the wealth of information at the molecular level, quantitative understanding of how bacteria perceive the state of cell growth is lacking. Here we reveal the underlying signaling strategies employed by E. coli to perceive and respond to growth, established through a series of physiological studies in which ppGpp and other key physiological variables are quantitatively measured.

During environmental changes such as diauxic shifts, E. coli responds by producing ppGpp ${ }^{23}$. Fig. 1a shows a typical diauxic growth curve in minimal medium containing glycerol and a small amount of glucose as the only carbon sources: cells grow exponentially on glucose without utilizing glycerol until glucose is depleted ${ }^{24}$, followed by a period of growth arrest (approximately 40-50 min in this case), before fully resuming growth on glycerol. We followed the kinetics of ppGpp accumulation by performing such growth transition experiments in the presence of ${ }^{32} \mathrm{P}$ - 
orthophosphate. Throughout the transition, labelled nucleotides were extracted and resolved by thin-layer chromatography (Fig. 1b). The ppGpp level relative to that of steady-state growth in glucose, denoted as $g(t)$, increased by over 8-fold within the first $10 \mathrm{~min}$ of glucose depletion before relaxing to a new steady-state level (Fig. 1c).

We also characterized changes in the translational elongation rate (ER, denoted by $\varepsilon$ ) during the growth recovery period by assaying for the delays in LacZ induction, as previous studies have established that ER determined from LacZ is representative of the ER of typical proteins ${ }^{25,26}$, and single molecule study of translation kinetics in vivo suggested little variation in ER in the absence of antibiotics ${ }^{27,28}$. Using the induction time obtained at various time $t$ (Extended Data Fig. 1a-c), the instantaneous ER, $\varepsilon(t)$, was deduced throughout the transition period (Fig. 1d, Extended Data Fig. 1d). The data shows an abrupt drop in ER immediately following glucose depletion and a gradual recovery before growth resumed. During the period of growth arrest, the time course of $\varepsilon(t)$ strikingly mirrored that of the relative ppGpp level $g(t)$ (Fig. 1c). Scatter plot of the ppGpp level with the reciprocal of ER exhibits a striking linear relation (Extended Data Fig. 1e). Defining the value of the extrapolated ER at $g=0$ to be $\varepsilon_{\max }$, the maximum elongation rate (to be justified below), the empirical relation between the relative ppGpp level and ER can be expressed as

$$
g=c \cdot\left(\frac{\varepsilon_{\max }}{\varepsilon}-1\right)
$$

where $\varepsilon_{\max } \approx 19.4 \mathrm{aa} / \mathrm{s}$ and $c \approx 4.6$; see Fig. $1 \mathrm{e}$.

The relation (1) between the ppGpp level and the translational elongation rate emerges straightforwardly from a model where the synthesis rate of ppGpp is proportional to the time the ribosome dwells on the A-site waiting for the cognate charged tRNA (Box 1a), and the latter can be rationalized molecularly based on the known structure of the ppGpp synthetase RelA in complex with the ribosome ${ }^{29,30}$ : As illustrated in Box 1b, RelA is activated (i.e., synthesizing ppGpp) only when it is complexed with an uncharged tRNA together at the A-site. This intricate design of RelA activation thus provides a way to probe the ribosome dwell-time on a codon, through the probability for RelA and uncharged tRNA to occupy the A-site. Assuming the synthesis of ppGpp is proportional to the occupation probability, these pieces suggest a mechanism for cells to detect the average dwell-time and encode it in the ppGpp level. Consistent with this idea, deletion of relA disrupts the linear relationship between ppGpp and ER (Extended Data Fig. 2).

Since the ppGpp level and ER are both known to change with the cellular growth rate ${ }^{25,31}$, we further examined their mutual relationship during steady-state growth. We grew E. coli with different nutrient composition at growth rates ranging from $0.13 \mathrm{~h}^{-1}$ to $0.96 \mathrm{~h}^{-1}$ (Supplementary Table 1) and measured the steady-state ppGpp levels relative to that in glucose, as well as the corresponding translational elongation rates; see Methods. As growth rate was reduced, ppGpp levels increased while ER decreased, (Fig. 2a, 2b, red squares), consistent with earlier reports ${ }^{25,31}$ Additionally, ER has recently been shown to increase in the presence of sub-lethal amounts of chloramphenicol $(\mathrm{Cm})^{25}$. Accordingly, we observed ppGpp levels to decrease and ER to increase during steady-state growth in the presence of increasing doses of $\mathrm{Cm}$ (Fig. 2a, 2b, green triangles). Owing to the difficulty of detecting low ppGpp levels, we used a $\Delta p t s G$ strain (NQ1261) ${ }^{25}$ which 
has reduced glucose intake and thereby shows elevated ppGpp levels in the absence of Cm. This strain allowed us to quantify changes in ppGpp level and compare it to the changes in ER under $\mathrm{Cm}$ treatment. Scatter plot of the steady state ppGpp level with the reciprocal of ER under carbon limitation again exhibited a linear relation (red squares, Fig. 2c). Moreover, those from Cminhibited cells fell on the same linear relationship (green triangles). Strikingly, this is the same relationship as the one observed during the diauxic shift (compare with blue circles in Fig. 2d), i.e., Eq. (1) with the same intercept and proportionality constant.

A steady state relationship between ER and ppGpp allows the cell to link ppGpp uniquely to the steady state growth rate via a simple regulatory circuit (Box 2): Due to negligible rate of protein turnover $^{32,33}$, the rate of protein synthesis is given by the product of ER and the number of active ribosomes per cell, $N_{R}^{a c t}$. During exponential growth at rate $\lambda$, the total peptide synthesis rate is $\lambda \cdot M_{P}$, where $M_{P}$ is the total protein mass per cell (in unit of the mass of an amino acid). The number of active ribosomes is the difference between the total number of ribosomes per cell $\left(N_{R}\right)$ and the number of inactive ribosomes per cell $\left(N_{R}^{\text {inact }}\right)$. Thus,

$$
\lambda M_{P}=\varepsilon \cdot\left(N_{R}-N_{R}^{\text {inact }}\right)
$$

The ratio $N_{R} / M_{P}$, which is proportional to the cellular ribosome concentration (since $M_{P}$ is proportional to the cell volume ${ }^{34}$ ), is set by the ppGpp level through regulation of rRNA expression $^{6}$. We take this regulatory function to be

$$
N_{R} / M_{P} \equiv R(g)=a / g
$$

(with an unknown constant $a$ ) since the RNA/protein mass ratio, which is proportional to $R$, scales as $1 / g$; see Fig. 3a. The inactive ribosome concentration, which is proportional to $N_{R}^{\text {inact }} / M_{P}$, is difficult to define and quantify directly. However, since it arises due to the binding of ribosomes to various ribosome hibernation factors, e.g., Rmf, Hpf, and RaiA ${ }^{35-37}$, and the abundances of the latter proteins all increase linearly with the ppGpp level as growth rate is reduced by limiting carbon uptake (Extended Data Fig. 3), we assume the form

$$
N_{R}^{\text {inact }} / M_{P} \equiv H(g)=b \cdot g
$$

for simplicity, with another unknown constant $b$.

Putting together the form of the regulatory factors in Eqs. (3) and (4) into Eq. (2) leads us to a relationship between the growth rate and the ppGpp level for exponentially growing cells:

$$
\lambda=\varepsilon(g) \cdot(R(g)-H(g))=\varepsilon(g) \cdot\left(\frac{a}{g}-b \cdot g\right) .
$$

where $\varepsilon(g)=\varepsilon_{\max } /(1+g / c)$ is obtained by inverting Eq. (1). The two constants $a$ and $b$ in Eq. (5) specify the magnitudes of the two regulatory interactions. With appropriate choices of these two constants, the simple model defined by Eq. (5) is able to quantitatively capture all the observed 
correlations among the growth rate $\lambda$, the ppGpp level $(g)$, the ribosome content $(R)$, and the elongation rate $(\varepsilon)$ (Fig. $3 \mathrm{~b}$-e), with model predictions based on best-fitted values of $a$ and $b$ shown as solid lines. In particular, the model recapitulated the well-known inverse relation between the growth rate and ppGpp level (Fig. 3b) ${ }^{31,38}$. This illustrates the general principle that the cell can perceive its own growth rate by incorporating the sensing of ER (Eq. (1)) into a simple regulatory circuit (Box 2) that controls the active ribosome content by the sensor. Equally importantly, the simple correspondence between the growth rate and ppGpp level enables the cell to implement growth-rate dependent control of many cellular functions, ranging from metabolism to cell division control, by simply using ppGpp to control the expression of the relevant genes $3,18,21,39,40$. In a previous study on bacterial growth control ${ }^{41}$, an ansatz was introduced in which the translational activity $(\sigma \equiv \lambda / R)$ was used to control gene expression during diauxic shifts. The results here establish a one-to-one relation between $\sigma$ and $g$ (Extended Data Fig. 4), thereby providing a mechanistic justification to the ansatz used in Ref. ${ }^{41}$.

At a quantitative level, our model captured the approximate linear relation between the ribosome content and the growth rate (Fig. 3c), the celebrated growth law discovered long ago by Schaecter et $\mathrm{a}^{10,42}$. Additionally, the model captured the Michaelis-like relation between the ER and the ribosome content (Fig. 3d), substantiated with extensive data collected from many conditions as reported in Dai et $\mathrm{al}^{25}$. Notably, a fit of the data to the Michaelis-Menten relation (Extended Data Fig. 5) recovers a maximum elongation rate $(20.0 \pm 1.9 \mathrm{aa} / \mathrm{s})$ that is indistinguishable from $\varepsilon_{\max }=19.4 \pm 1.4 \mathrm{aa} / \mathrm{s}$ defined by taking $g \rightarrow 0$ in Eq. (1) (Extended Data Fig. 1e). Finally, the model captures the weak dependence of the elongation rate at different growth rate (Fig. 3e), with the minimal ER in slow growth condition, denoted by $\varepsilon_{\min }$, being close to one-half of $\varepsilon_{\max }$.

The emergence of a simple linear relation between the ribosome content $R$ and the growth rate $\lambda$ is surprising, given the nonlinear regulatory effects exerted by ppGpp (Eq. (5)). In fact, a variety of relationships among these quantities is possible for generic values of $a$ and $b$ (Extended Data Fig. 6). However, if the regulatory parameters $a$ and $b$ are such that the ratio $\varepsilon_{\text {min }}: \varepsilon_{\max }$, is exactly one-half, then mathematically the model yields an exact linear relation between $R$ and $\lambda$, with the slope given by $1 / \varepsilon_{\max }$, and an exact Michaelis-Menten relation between the ER and the ribosome content, with the maximal ER being $\varepsilon_{\max }$ (Supplementary Note). Thus, to the extent that Eqs. (3) and (4) capture the forms of the regulatory functions, prescribing the appropriate regulatory parameters $a$ and $b$ to enforce $\varepsilon_{\min }$ being approximately one-half of $\varepsilon_{\max }$ is crucial for the emergence of the approximate linear growth relation between the ribosome content and the growth rate. (We have separately shown that adding offsets to the simplest forms of the regulatory functions used in Eqs. (3) and (4) do not affect the quality of the fit; see Extended Data Fig. 7.)

Maintenance of ER above a minimal level is clearly of physiological importance, as too low an ER would lead to problems in the processivity of protein synthesis ${ }^{43,44}$. Another physiological requirement is the maintenance of a sufficient ribosome reserve at slow growth, denoted by $R_{0}$, needed for rapid growth recovery when favorable nutrient conditions return ${ }^{34}$. Both physiological requirements are satisfied by employing hibernation factors to inactivate ribosomes. By employing both positive and negative regulation through distinct promoters (Box 2), the cell can readily attain the required values of $\varepsilon_{\min }$ and $R_{0}$ by simply prescribing the regulatory parameters $a$ and $b$; see Extended Data Fig. 8 and Supplementary Note. To keep $\varepsilon_{\text {min }}$ high while also maintaining a ribosome reserve is possible in principle; see Extended Data Fig. 9. With high ER (e.g., above 
90\% of $\varepsilon_{\max }$, dashed line in Extended Data Fig. 9a), the ribosome content would even be moderately reduced at fast growth rate (Extended Data Fig. 9b), a fitness benefit from the proteome allocation perspective ${ }^{12,45}$. However, this strategy would also require significant improvement in detecting very short translational dwell time (right axis, Extended Data Fig. 9d). Thus, the choice of using $\varepsilon_{\min } \approx \varepsilon_{\max } / 2$ may reflect a compromise between the physiological demand for keeping $\varepsilon_{\min }$ high and the molecular constraint for detecting small changes in ER in order to sense slow growth and enforce growth-rate dependent regulation.

Regarding the mechanism of ER sensing in the steady state, it has been long known that RelA is not essential during steady-state growth ${ }^{31}$. Indeed, ppGpp levels and elongation rates in the $\Delta$ relA strain are similar to that of the wild-type (Extended Data Fig. 10a,b) and the ppGpp-ER relationship is maintained in the $\triangle$ relA strain under steady-state growth (Extended Data Fig. 10c, black symbols), even though this relationship was disrupted during the diauxic shift for the $\Delta$ relA

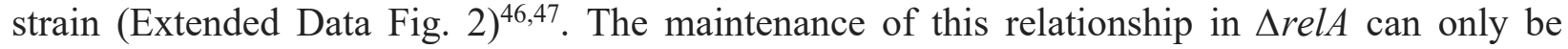
attributed to SpoT (the other ppGpp synthetase in E. coli ${ }^{8,17}$ ), although how SpoT may sense ER is not known. While there is some evidence of SpoT associated with the ribosome ${ }^{48}$, the functional significance of this association is unclear. There is always the possibility that an unknown mediator which interacts with the ribosomes in way analogous to RelA (Box 1b) may affect the activity of SpoT. Alternatively, given the Michaelis-like relation between the ribosomal content and the ER in steady state (Fig. 3d), interaction of SpoT with an entity co-regulated with the ribosome (e.g., uncharged tRNA ${ }^{49,50}$ ) may be sufficient to convey ER to SpoT in steady state conditions.

Cell growth is an emerging property of a living cell that involves the coordinated dynamics of many thousands of molecular species and processes. Accurately perceiving the state of cell growth is crucial for cellular decision making but is a challenging task for molecular signaling. E. coli accomplishes this important task not based on concentrations of molecules, but rather, based on the rate of translational elongation. Because translational elongation is affected by many metabolic processes in the cell (Box 2), monitoring the elongation rate is an effective strategy to diagnose the state of cell growth independently of specific nutrient bottlenecks. Here lies the mechanistic origin of the generality of the phenomenological growth laws, i.e., why the same quantitative relation between the ribosome content and the growth rate is sustained regardless of whether cell growth is limited by carbon supply ${ }^{10,12,13}$, nitrogen supply ${ }^{51}$, partial auxotrophy ${ }^{2,13}$, or drugs which inhibits tRNA charging ${ }^{52}$. This mechanism also predicts generally that for perturbations not captured by the translational dwell-time, including antibiotics ${ }^{25,53}$, phosphate limitation ${ }^{51}$ and lipid stress $^{54}$, growth rate perception is distorted and the growth law of Schaecter et al ${ }^{10}$ is altered.

Sensing of the translational elongation rate, which underlies E. coli's ability to reduce the highdimensional complexity of the metabolic state of a cell down to a central signal, the ppGpp level, provides a rare, trackable example of how cells perform dimensional reduction at the molecular level to attain crucial physiological information at the cellular level ${ }^{55}$. Identifying and elucidating further instances of such strategies of dimensional reduction employed by cells will be instrumental in fundamentally advancing our understanding of the connection between molecular interaction and cellular physiology for prokaryotes as well as for eukaryotes. 


\section{Methods and Materials}

\section{Growth media composition and culture conditions}

Steady-state and growth transitioning cultures were grown in MOPS based minimal media ${ }^{56}$ supplemented with various carbon sources and chloramphenicol as indicated in Supplementary Table 1 . All cultures were grown at $37^{\circ} \mathrm{C}$ with shaking at $250 \mathrm{rpm}$. For every experiment, culturing was carried out through sequential propagation of seed cultures in LB, pre-cultures in the experimental medium, and the experimental cultures. Single colonies from fresh LB agar plates were first grown in LB broth for $6 \mathrm{hrs}$ as the "seeding culture". In the pre-culturing step, depending on the experiment, cells from seeding cultures were diluted into appropriate media such that the pre-cultures would still be in exponential growth phase after overnight growth. Media used for pre-culturing in steady-state experiments were same as the experimental media (Supplementary Table 1). For the glucose to glycerol transitions, optical density was monitored at $600 \mathrm{~nm}\left(\mathrm{OD}_{600}\right)$ to follow the growth transition kinetics. Pre-cultures were grown in medium supplemented with $20 \mathrm{mM}$ glucose and $20 \mathrm{mM}$ glycerol, to avoid glucose run-out during the pre-culturing step. Exponentially growing pre-cultures were then diluted in the appropriate experimental medium (pre-warmed) at an initial $\mathrm{OD}_{600}$ of $\sim 0.005$ and various measurements were carried in the $\mathrm{OD}_{600}$ range of $0.1-0.4$.

\section{Strain construction}

Wild type E. coli $\mathrm{K}-12$ NCM3722 $2^{57,58}$ and its derivatives were used in this work. HE838 (ArelA) was constructed using the $\lambda$-Red recombinase method $^{59}$ as follows. The km resistance gene was amplified from pKD13 using chimeric oligos relA1-P1 and relA2-P2 (Supplementary Table 2). The PCR products were electroporated into NCM3722 cells expressing Lambada-Red proteins encoded by pKD46. The Km resistant colonies were confirmed by PCR and sequencing for the replacement of the region harboring $\mathrm{relA}$ by the $\mathrm{km}$ gene.

\section{Translation elongation rate (ER) measurement}

ER was measured using LacZ as a reporter as described in Dai et al ${ }^{25}$ with modifications. Depending on the experiment, $10 \mathrm{ml}$ cultures were either grown in different steady state conditions or as undergoing glucose to glycerol growth-transition. When cultures reached $\mathrm{OD}_{600}=0.4$ (for steady-state growth) or at specific time-points during the growth transition, $5 \mathrm{mM}$ isopropyl- $\beta$-Dthiogalactoside (IPTG) was added to induce the lac operon. Immediately after induction, $500 \mathrm{ml}$ samples were taken at $10 \mathrm{~s}$ or $15 \mathrm{~s}$ intervals to pre-cooled $\left(-20^{\circ} \mathrm{C}\right)$ tubes with $20 \mathrm{ml}$ of $0.1 \mathrm{M}$ chloramphenicol and then rapidly frozen by dry ice. Samples were stored at $-80^{\circ} \mathrm{C}$ before betagalactosidase assay. 4-methylumbelliferyl-D-galactopyranoside (MUG, a sensitive fluorescence substrate) was used to measure LacZ activities in this work. Briefly, each sample was diluted by Z-buffer by 5-fold and added to 96 -well plate to a volume of $200 \mathrm{ml}$. Plate was warmed at $37^{\circ} \mathrm{C}$ for 10 mins before adding MUG. Tecan (SPARK) plate reader was used for MUG injection and fluorescence readings. $20 \mathrm{ml}$ of $2 \mathrm{mg} / \mathrm{ml} \mathrm{MUG}$ was injected to each well and fluorescence intensity (365nm excitation filter, $450 \mathrm{~nm}$ emission filter) was measured every 4 mins for 2 hours. In the linear range of fluorescence intensity vs time plot, a linear fit was applied to obtain the slope as the relative LacZ activity for each sample. By plotting the square root of the relative LacZ activity above basal level against time ${ }^{60}$ (Schleif plot), the lag time for the synthesis of the first LacZ molecule $\left(T_{\text {first }}\right)$ was obtained for each sample; see Extended Data Fig. 1a-c). The ER measured 
in this work was found to be slightly higher than that reported by Dai et $\mathrm{al}^{25}$, likely due to the higher sensitivity of the substrate MUG compared to ONPG as used previously ${ }^{25}$.

\section{ppGpp measurement}

ppGpp measurements were carried out as described by Cashel ${ }^{61}$ with minor modifications. Typically, experimental cultures were grown in $3 \mathrm{ml}$ volumes. Labelling was carried out when the experimental cultures grew to $\mathrm{OD}_{600}=0.02$ by adding $0.1 \mathrm{mCi}{ }^{32} \mathrm{P}$-orthophosphate (Perkin Elmer) per ml culture. For steady-state growth, $20 \mu \mathrm{l}$ aliquots were drawn at various $\mathrm{OD}_{600}$ values between the range 0.1-0.4 (see Supplementary Fig. 1a), and added to an equal volume of pre-chilled 10\% formic acid. For cultures undergoing diauxic shift, $20 \mu \mathrm{l}$ aliquots were drawn at various time points during the transition, and added to an equal volume of pre-chilled $10 \%$ formic acid. Formic acidextracts were spun down at $13 \mathrm{k}$ rpm for 10 minutes and a total of $2 \mu \mathrm{l}$ supernatant was spotted $0.5 \mu \mathrm{l}$ at a time near the base of a PEI-Cellulose $\mathrm{F}$ thin layer chromatography plate (Millipore). The spots were dried and nucleotides were resolved using freshly prepared $1.5 \mathrm{M} \mathrm{KH}_{2} \mathrm{PO}_{4}(\mathrm{pH} 3.4)$. The TLC plates were dried and exposed to a phosphorimaging screen for 24-36 hours. Chromatograms were imaged using a Typhoon FLA 9500 scanner (GE) and analyzed using Fiji software. For steady state growth conditions, the slope of ppGpp signal intensities versus OD600 were compared among different cultures to obtain the relative ppGpp levels, as shown in Supplementary Fig. 1c. For any batch of measurements, the ppGpp level from a sample of NCM3722 growing steadily in MOPS glucose was always included as a reference. All measurements in that batch were normalized to the glucose-grown reference in the same batch.

\section{Total RNA and Protein measurement}

Total RNA was measured using the method of Benthin et $\mathrm{al}^{62}$, and protein was measured using the Biuret method ${ }^{63}$, with minor modifications as described by You et $\mathrm{al}^{13}$.

\section{Acknowledgements}

We are grateful to technical advices from Xiongfeng Dai and Manlu Zhu in early stages of this work and helpful discussions on the general issue of biological dimension reduction with Boris Shraiman. This work was supported by the NSF through Grant MCB-1818384 and the NIH through Grant GM109069. 
a

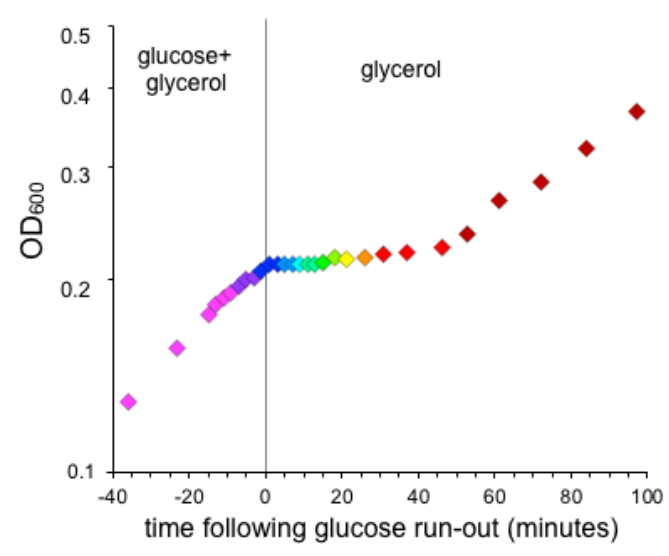

b

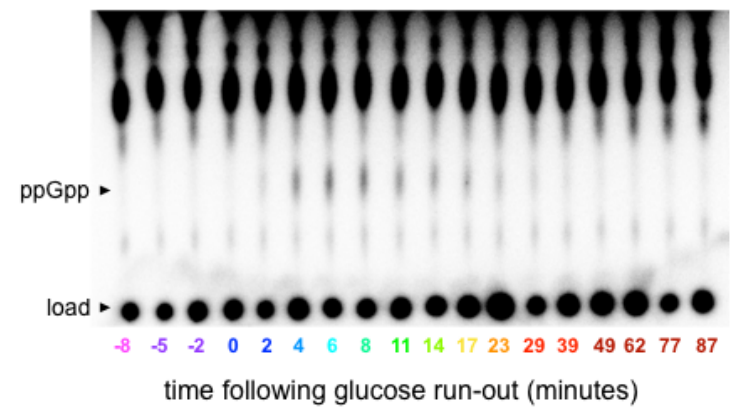

d
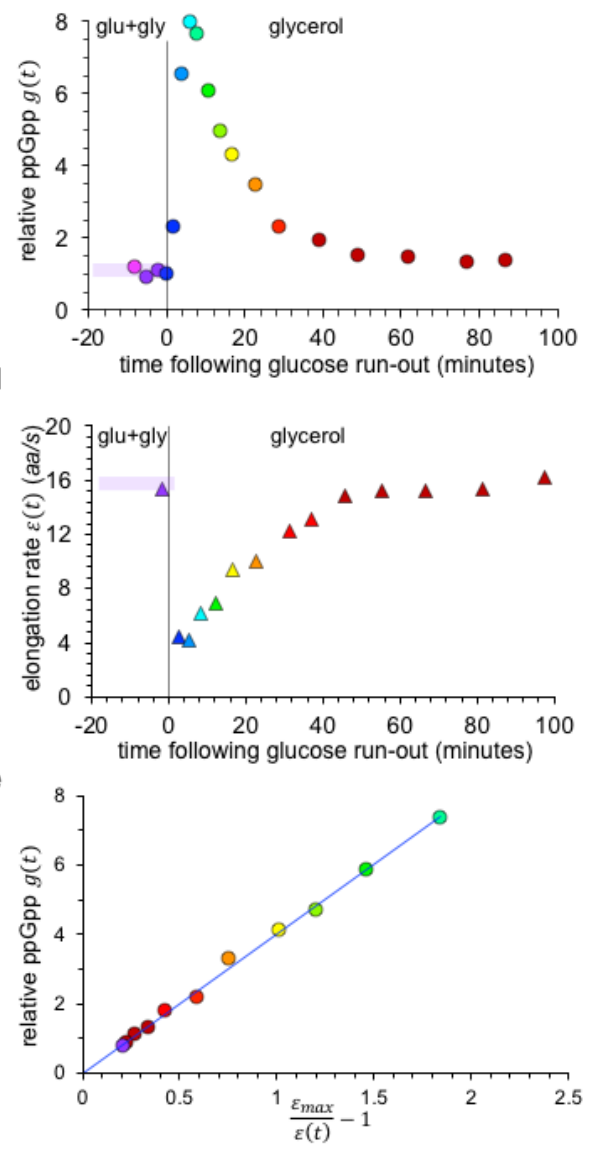

Figure 1: Relation between ppGpp and the translational elongation rate during growth transition. a, Growth kinetics of E. coli K-12 NCM3722 monitored by measuring the optical density at $600 \mathrm{~nm}\left(\mathrm{OD}_{600}\right)$ during the diauxic transition from glucose to glycerol. The same colorscheme is used across the panels to match different measured quantities to samples taken at different time during the growth transition. $\mathbf{b}$, Resolution of ppGpp in cells sampled at different time during the growth transition by thin layer chromatography (TLC). The spots at bottome correspond to sample loading and the migrated ppGpp are indicated alongside the chromatogram. Signal intensity from the chromatograph was used as the measure of the ppGpp level. c, Signal intensity for ppGpp obtained from panel $b$ is normalized by that before the growth shift, denoted by $g(t)$, is plotted against the time $t$ at which the sample was taken. $\mathbf{d}$, The translational elongation rate $\varepsilon(t)$ was obtained as described in Extended Data Fig. 1, and plotted against the time $t$ at which sample was taken. e, ppGpp levels $g(t)$ are plotted against the reciprocal of the corresponding elongation rates normalized by the maximum elongation rate $\left(\varepsilon_{\max }\right)$, defined as the value of the elongation rate extrapolated to $g=0$ (see Extended Data Fig. 1d). The line is the best-fit of the data to Eq. (1), with $c \approx 4.6$ and $\varepsilon_{\max }=19.4 \pm 1.4 \mathrm{aa} / \mathrm{s}$. 

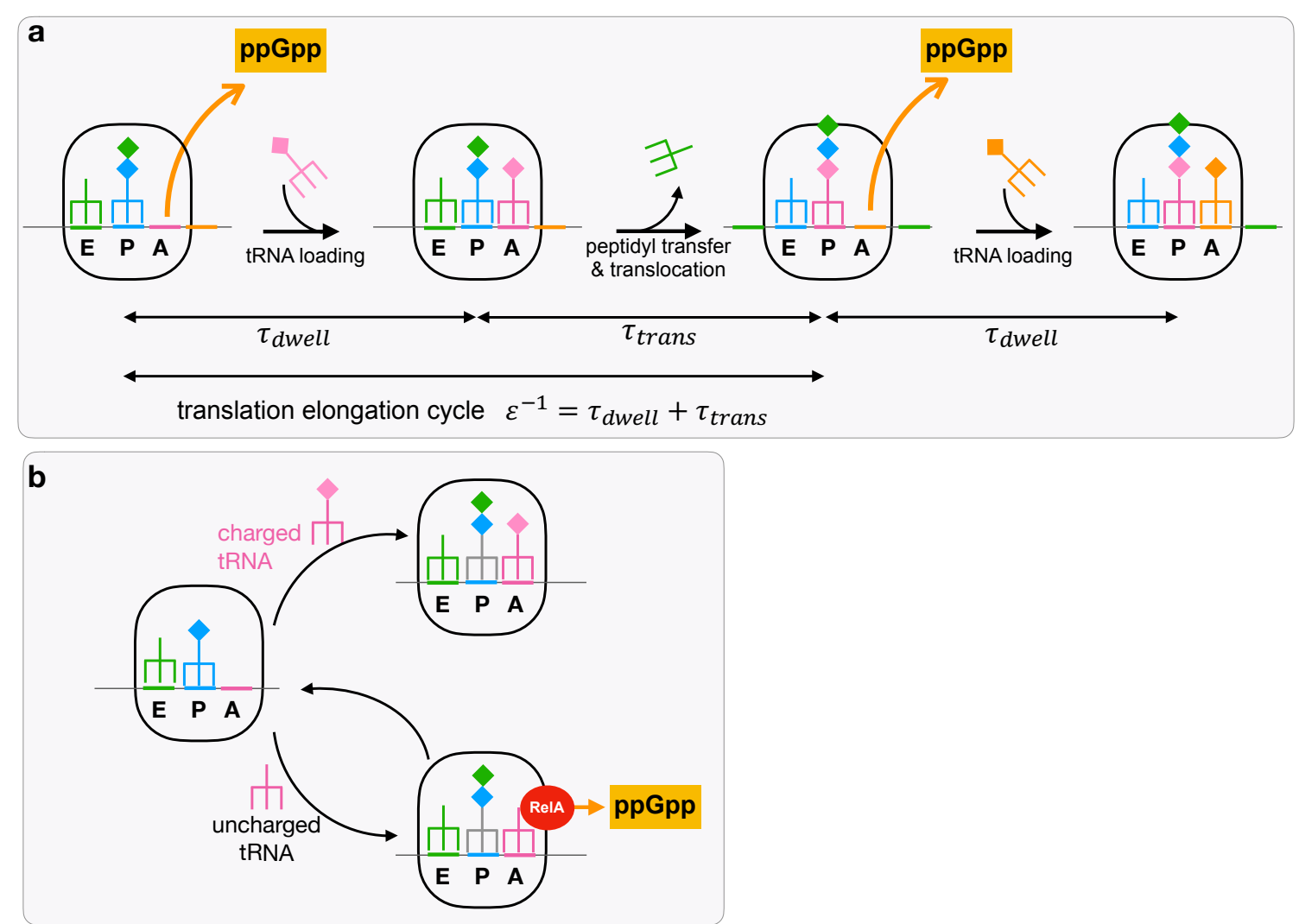

Box 1: Sensing of the translational elongation rate by ppGpp. a, A cycle of translation elongation constitutes the loading of a cognate tRNA to the A site (taking time $\tau_{d w e l l}$ ), followed by peptidyl transfer and the translocation of mRNA/tRNA (taking time $\tau_{\text {trans }}$ ). The total time for one cycle, given by the reciprocal of the translational elongation rate $\varepsilon$, is thus given by $\varepsilon^{-1}=$ $\tau_{\text {dwell }}+\tau_{\text {trans }}$. As the dwell time depends on the concentration of the charged tRNAs and hence the nutrient status, the fastest elongation speed is obtained when $\tau_{d w e l l} \rightarrow 0$, in which case $\varepsilon \rightarrow$ $\tau_{\text {trans }}^{-1} \equiv \varepsilon_{\text {max }}$. The empirical relation observed between ppGpp and the elongation rate $\varepsilon$, Eq. (1), can thus be alternatively written as $g=c \tau_{d w e l l} / \tau_{\text {trans }}$. Such a relation can result from a simple model where the rate of ppGpp synthesis is proportional to the dwell time, with a constitutive rate of ppGpp hydrolysis, i.e., $\frac{d g}{d t}=\alpha-\beta \cdot g$, where $\alpha \propto \tau_{d w e l l}$ and $\beta$ is constant. [Note that $\tau_{\text {trans }}$ or $\varepsilon_{\max }$ is not affected ${ }^{25}$ in the perturbations probed in this study.] b, According to biochemical and structural studies ${ }^{17,29,30,64}$, ppGpp synthesis is activated when the A-site of the ribosome is loaded by a RelA-bound uncharged tRNA. In rich nutrient conditions where charged tRNAs are abundant, the dwell time is short and RelA activity is low (top arrow). High levels of uncharged tRNAs, as expected in nutrient down-shifts, will prolong the dwell-time and lead to a corresponding increase in RelA-mediated ppGpp synthesis (bottom arrow). Thus, the design of RelA function allows the several hundred RelA molecules in a cell ${ }^{65}$ to sample the average dwelltime of the ribosomes on the A-site, and thereby monitor the overall nutrient status of the cell. 
a

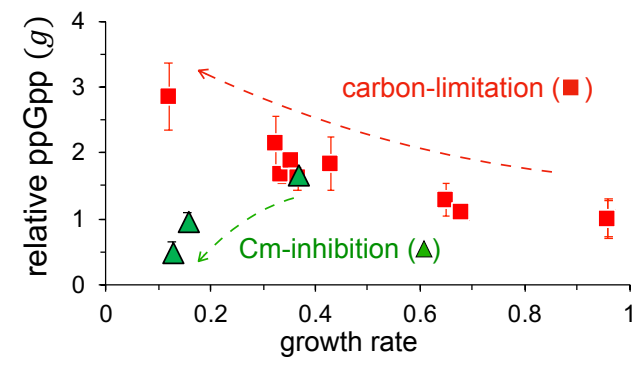

b

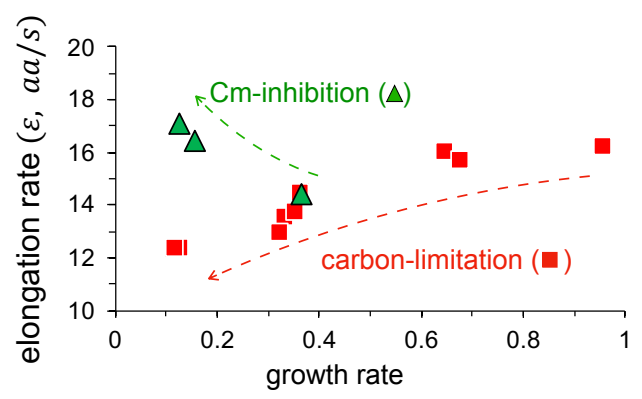

C

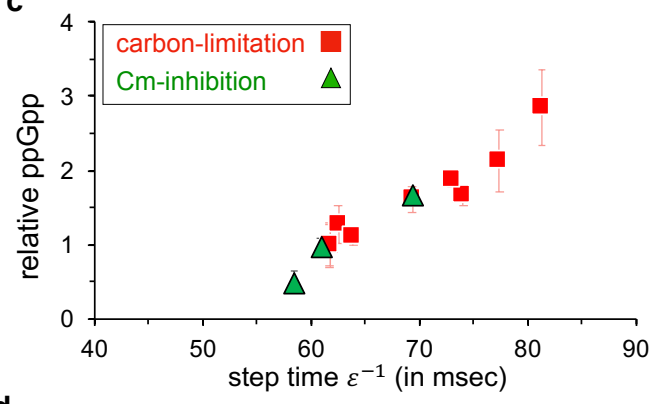

d

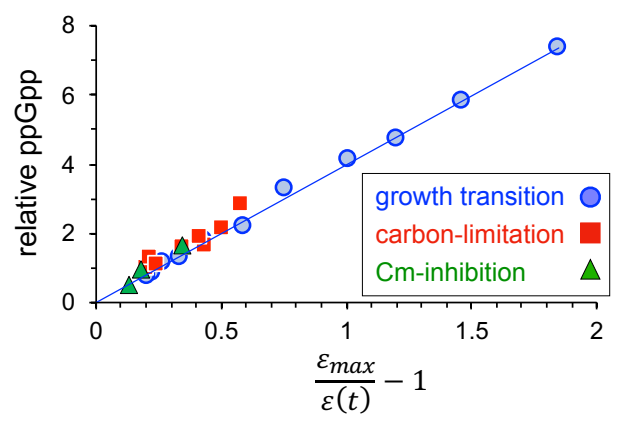

Figure 2: Relation between ppGpp and translational elongation rate during steady-state growth. a, ppGpp levels relative to that in the glucose minimal medium, $g$, are plotted against the corresponding growth rates for cells under steady state carbon-limited growth (red) and translation-limited growth (green) for cells treated with sub-lethal doses of chloramphenicol $(\mathrm{Cm})$; see Supplementary Table 1. For each growth medium, ppGpp level was obtained by measuring 4 samples taken from exponentially growing cells at different ODs and using linear regression (Methods and Supplementary Fig. 1). Error bars represent the uncertainty in the linear fit. b, Translation elongation rates are plotted against the steady state growth rates for carbon-limited (red) and Cm treated (green) cells. c, Scatter plot of reciprocal elongation rates (or the step time for ribosome advancement) in milliseconds and the relative ppGpp levels $(g)$ measured during steady-state growth for wild-type $E$. coli under carbon limitation (red) and translation inhibition (green). d, The same measurements from steady-state growth (panel c) are replotted by normalizing the elongation rate to $\varepsilon_{\max }$ together with the data collected under growth transition from Fig. 1e (blue symbols) for comparison. The line is the same as that in Fig. 1e. 


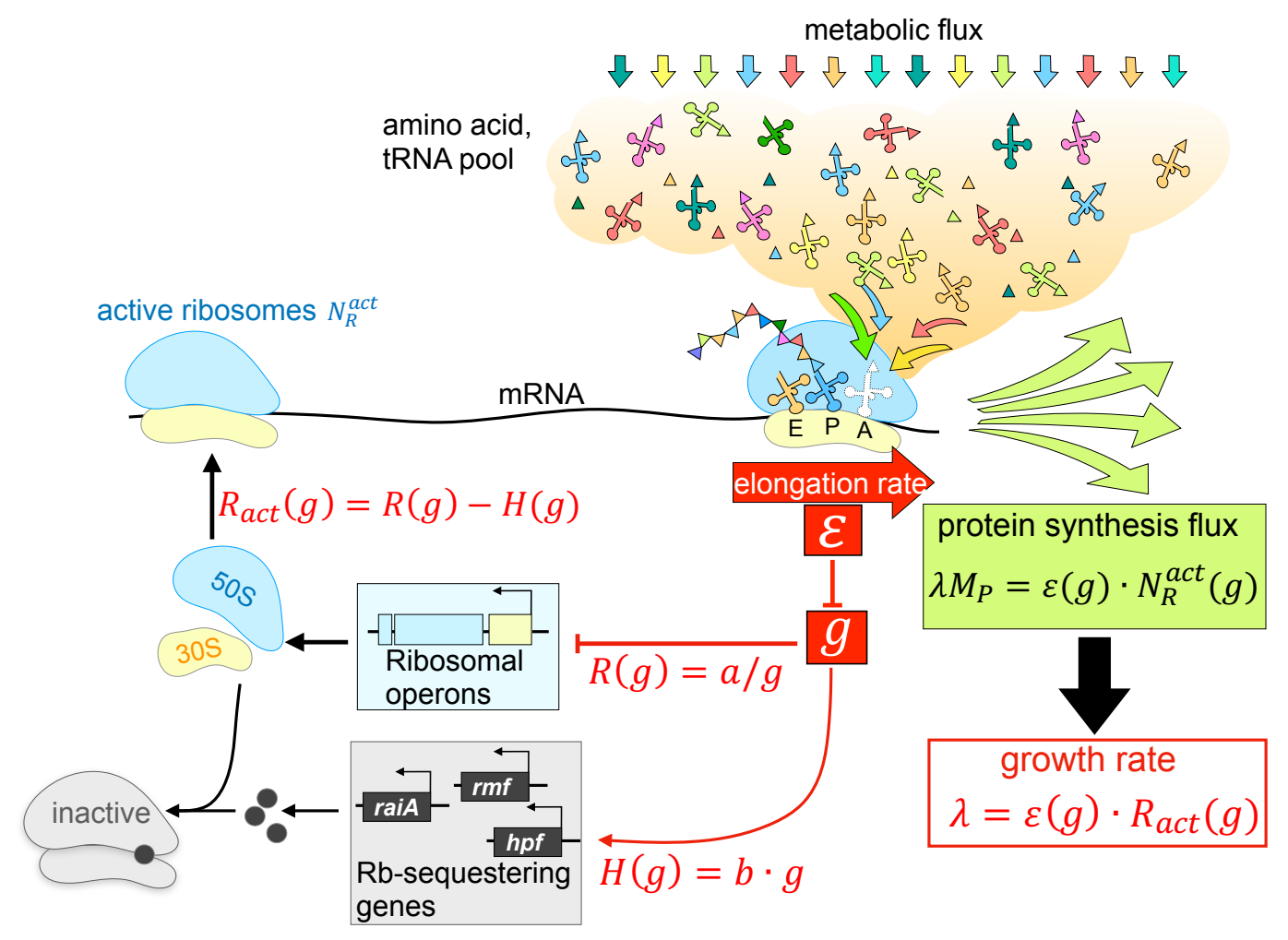

Box 2: Perception of cellular growth rate and dimensional reduction. Elongation rate-sensing by ppGpp synthesis (Box 1) allows the cell to relate the metabolic activity to cellular growth: The steady state protein synthesis flux $\lambda \cdot M_{P}$ is given by the product of the elongation rate $\varepsilon$ and the number of active ribosomes, $N_{R}^{a c t}$. Because $\varepsilon$ is a function of the ppGpp level $g$ (Fig. 1e), and the active ribosome content $R_{a c t} \equiv N_{R}^{a c t} / M_{P}$ is given by the difference between the total ribosome content $R(g)$ (Fig. 3a and Eq. (3)) and the content of the ribosome-sequestering elements $H(g)$ (Extended Data Fig. 3 and Eq. (4)), each of which is a function of $g$ due to ppGpp-mediated regulation, it follows that $\lambda=\varepsilon(g) \cdot R_{a c t}(g)$ is a function of $g$. Thus, the regulatory circuit arising from the sensing of ribosome activity and the regulation of active ribosome component by ppGpp allows the cell to perceive the cellular growth rate through the ppGpp level (Fig. 3b). Moreover, as charged tRNAs are delivered to the ribosomal A-site during translation, the dwelltime depends on the metabolic fluxes directed towards all the biosynthetic precursors needed for protein synthesis (the orange cloud in the illustration: triangles, amino acids; clover leaves, tRNA; colored arrows, fluxes). The translational elongation rate (ER) is therefore a single quantity that reflects the combined status of the hundreds of diverse metabolic reactions underlying protein synthesis and cell growth. In this sense, the mechanism of ER-sensing is a dimensional reduction technique employed by E. coli to detect a central cellular parameter, the growth rate, emergent from the hundreds of underlying metabolic reactions. The regulatory strategy depicted here provides the molecular basis of the phenomenological growth laws observed, as verified in Fig. 3c-e. 
a

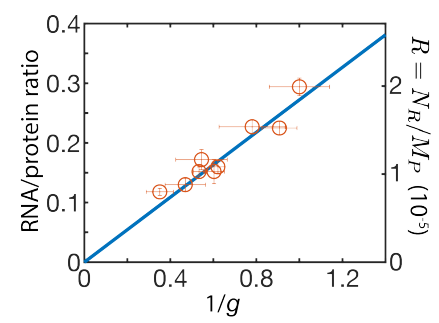

d

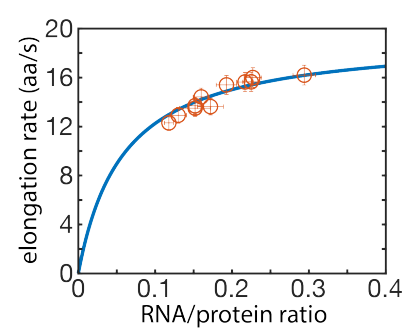

b

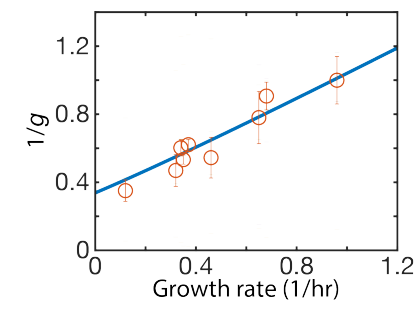

e

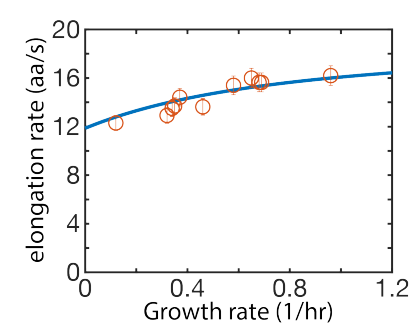

c

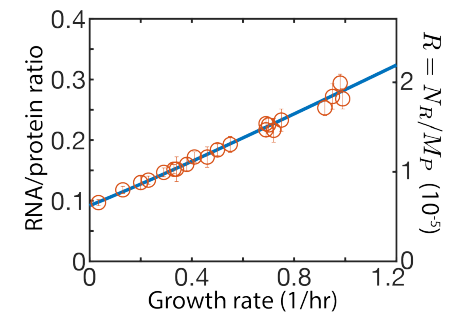

Figure 3. Model data comparison. a, RNA-protein ratio (circles, left vertical axis) is proportional to the reciprocal of ppGpp level. This ratio is taken to be proportional to the ribosome content, $N_{R} / M_{P}$ (right vertical axis), and is used as an input to the model; see Eq. (3). The proportionality constant between RNA-protein ratio and $N_{R} / M_{P}$, denoted by $\eta$, is one of the 3 fitting parameters of the model. The best-fit is shown by the line. b, The approximate linearity between the RNAprotein ratio and the growth rate (circles, left vertical axis) is well accounted for by the model (line), as is the approximate linear relation between the growth rate and the reciprocal of ppGpp level (panel c), and the weak relation between the ER and growth rate (panel d), and the Michaelis relation between ER and the RNA-protein ratio (panel e). The model is described by Eqs. (1) and (5). The values of the best-fit parameters were $a \cong 1.85 \times 10^{-5}, b \cong 2.11 \times 10^{-6}, \eta \approx$ $6.8 \times 10^{-5}$. 


\section{Extended Data}
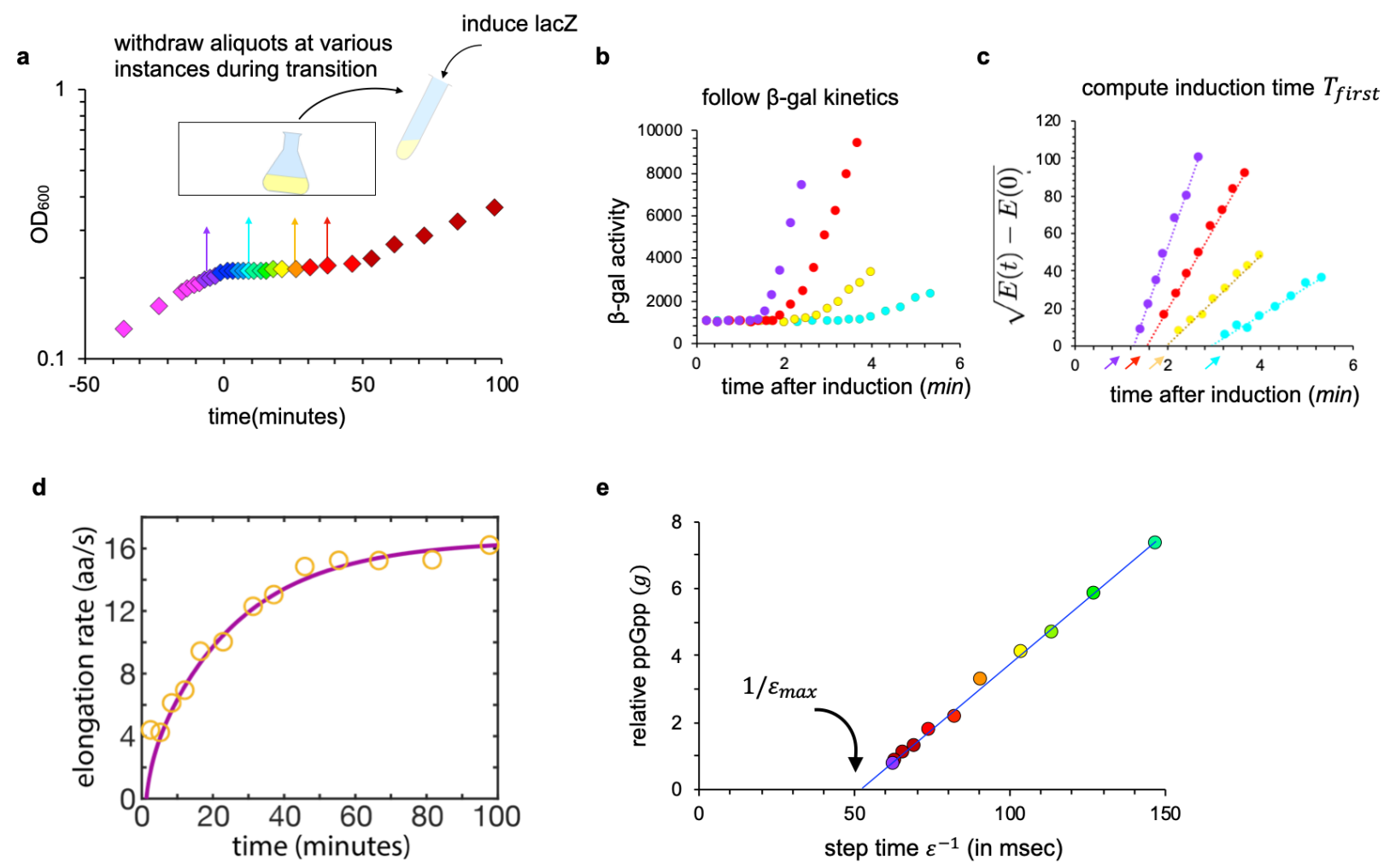

Extended Data Figure 1. Elongation rate measurements during diauxic shift. a, Scheme for measuring the instantaneous translation elongation rates $\varepsilon(t)$ during growth transition. At different time $(t)$ during the diauxic transition, $10 \mathrm{ml}$ aliquots were removed into a fresh tube and the synthesis of the reporter protein LacZ was induced immediately by the addition of IPTG. b, LacZ induction curves for the four samples taken (indicated by the arrows in panel a). LacZ induction kinetics of each sample was followed by monitoring the $\beta$-galactosidase activity using 4methylumbelliferyl-D-galactopyranoside (MUG) assay (see Methods and Materials for details). c, The square root of lacZ activity above basal level were plotted against induction time to obtain the lag time for the synthesis of the first LacZ molecule $\left(T_{\text {first }}\right.$, shown by the arrows $\left.{ }^{60}\right)$. The translational elongation rates shown in Fig. 1 were obtained as $\varepsilon=L_{\text {LacZ }} /\left(T_{\text {first }}-T_{\text {init }}\right)$ with $L_{\text {LacZ }}$ being the length of LacZ monomer (1024aa) and $T_{\text {init }}$ being the time taken for the initiation steps (10s across various nutrient conditions ${ }^{25}$ ). d, Comparison of the elongation rate obtained from the naïve approach described above with a more detailed calculation using all of the LacZ induction data together. The yellow circle represents the naïve calculation of ER at the various time where samples were taken. The purple line represents $\varepsilon(t)$ calculated from solving the following integral equation

$$
\int_{t_{i}+T_{\text {init }}}^{t_{i}+T_{\text {first }}\left(t_{i}\right)} \varepsilon(t) d t=\int_{0}^{t_{i}+T_{\text {first }}\left(t_{i}\right)} \varepsilon(t) d t-\int_{0}^{t_{i}+T_{\text {init }}} \varepsilon(t) d t=L_{\mathrm{LacZ}}
$$


where $t_{i}$ is the time when IPTG is added. $T_{\text {first }}\left(t_{i}\right)$ denotes the lag time obtained from the LacZ induction curve when IPTG was added at time $t_{i}$. $T_{\text {init }}$ was still taken to be as the same constant (10s). Taking the derivative of $t_{i}$ on both sides of Eq. (E1), we obtain

$$
\varepsilon\left(t_{i}+T_{\text {first }}\left(t_{i}\right)\right) \times\left(1+\frac{d T_{\text {first }}\left(t_{i}\right)}{d t_{i}}\right)=\varepsilon\left(t_{i}\right) .
$$

By fitting $T_{\text {first }}\left(t_{i}\right)$ to an exponential function $y=a_{1} \exp \left(a_{2} x\right)+a_{3}$, we estimated $\frac{d}{d t_{i}} T_{\text {first }}\left(t_{i}\right)$ at each time point during the shift. For $t_{i}>40 \mathrm{~min}$, since the change in $T_{\text {first }}$ is relatively small (circles in (d) represent the inverse of $\left.T_{\text {first }}\right)$, we Taylor-expanded $\varepsilon\left(t_{i}+T_{\text {first }}\left(t_{i}\right)\right.$ ) at $t_{i}$ and solved Eq. (E2) analytically with the boundary condition $\varepsilon(100 \mathrm{~min})=\varepsilon_{\text {glycerol }}=16 \mathrm{aa} / \mathrm{s}$. For $t_{i}<40 \mathrm{~min}$, we directly used Eq. (E2) to calculate $\varepsilon\left(t_{i}\right)$ from $\varepsilon\left(t_{i}+T_{\text {first }}\left(t_{i}\right)\right)$ at a later time numerically. Notice that the difference between the two ways of calculating the instantaneous ER is negligible. This is because the time scale of $T_{\text {first }}$, a few minutes as seen in panel (c), is overall much smaller than the time scale of the shift ( $\sim 40 \mathrm{~min})$. So we simply reported the result of the naive ER calculation through this work. e, Relative ppGpp level $g(t)$ obtained in Fig. 1c are plotted against the reciprocal of the elongation rate, or the step-time for ribosome advancement. $\varepsilon(t)$ values for the time points at which ppGpp levels were measured were estimated from fitting the naïve calculation of ER vs time in panel (d) to an exponential function. A linear fit of the data in panel (e) yields the x-intercept $1 / \varepsilon_{\max }$, with $\varepsilon_{\max }=19.4 \pm 1.4 \mathrm{aa} / \mathrm{s}$. 


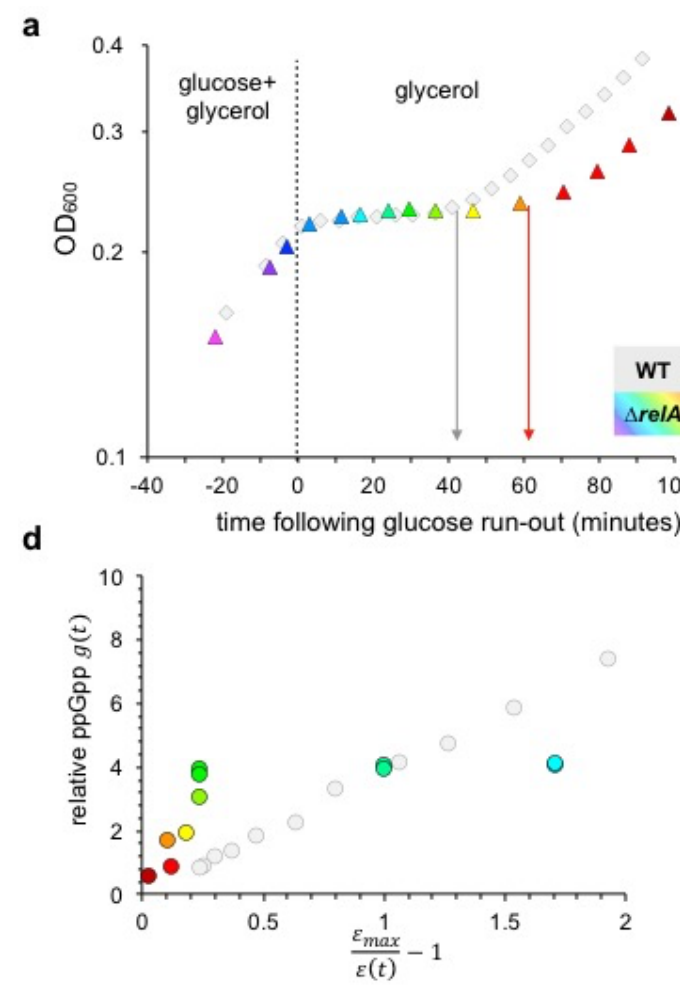

b

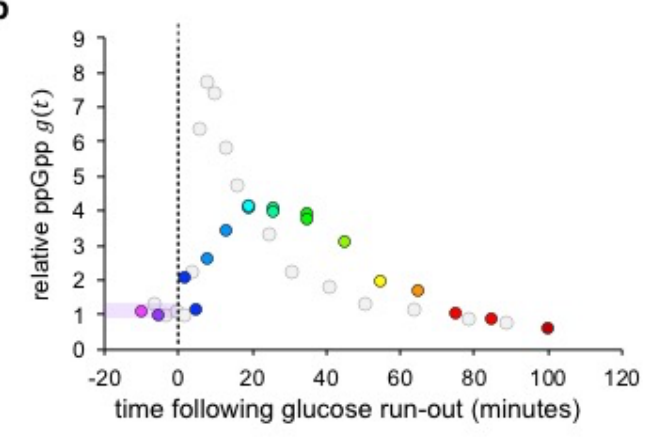

c

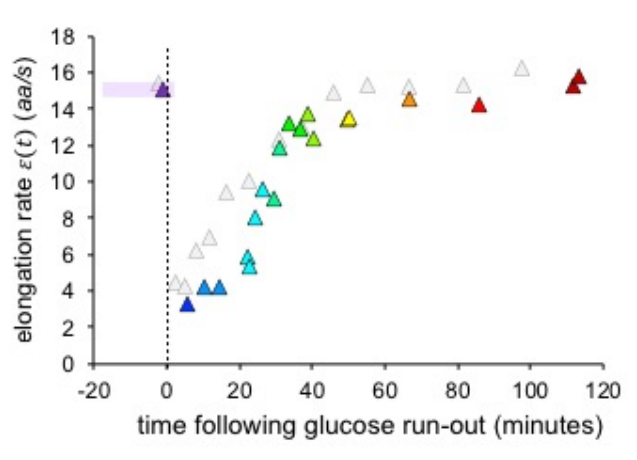

Extended Data Figure 2. Relation between the ppGpp level and elongation rate for the $\Delta$ relA strain during diauxic shift. a, Growth kinetics of the $\Delta$ relA strain (colored triangles) and the wild type (grey circles, same as in Fig. 1a) during the glucose-to-glycerol diauxic transition. Vertical arrows indicate the duration of the lag period following glucose depletion before growth resumes on glycerol. Color-scheme corresponding to individual samples for $\Delta$ relA is shared across panels in this figure. b, ppGpp levels measured for the $\Delta$ relA strain (colored) and the wild type (grey, same as those in Fig. 1c), relative to the WT ppGpp level in glucose minimal medium, during the glucose-to-glycerol transition. Note that pre-shift ppGpp levels of the two strains are indistinguishable. c, Instantaneous translation elongation rate measured for the $\Delta$ relA strain (colored) and the wild type (grey, same as those in Fig. 1d) during the glucose-to-glycerol transition. $\mathbf{d}$, The relation between ppGpp level and the instantaneous elongation rate for the $\Delta$ relA strain (colored) and the wild type (grey, same as those in Fig. 1e) during the glucose-to-glycerol transition. 
a

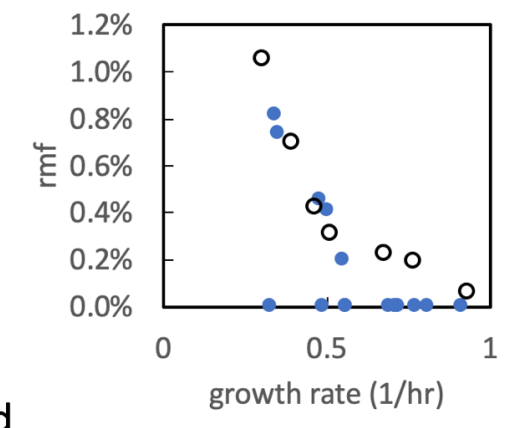

d

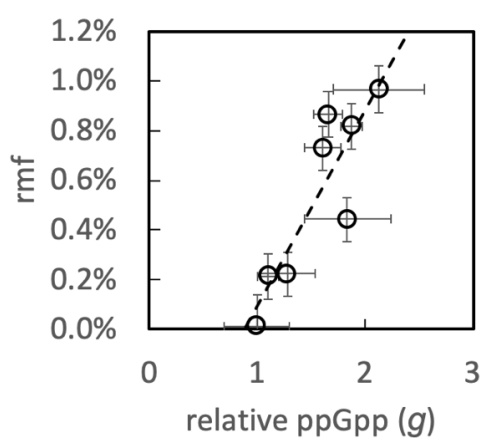

b

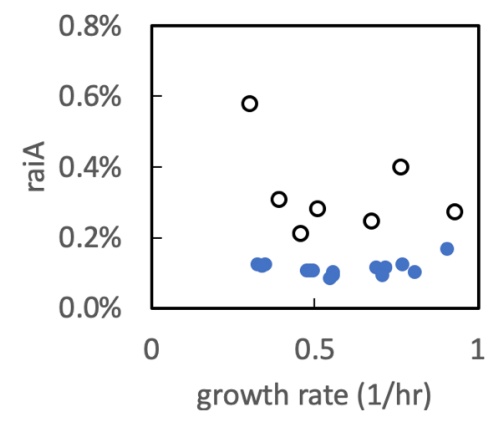

e

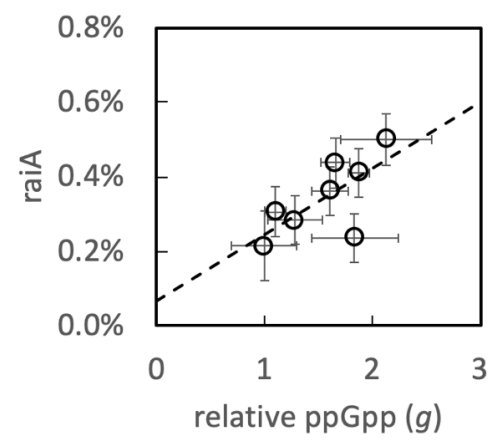

C
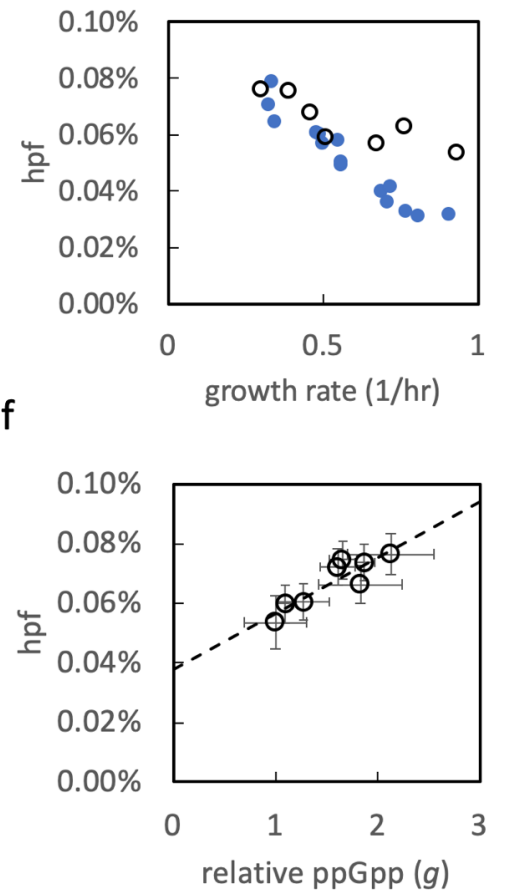

Extended Data Figure 3: Relation between the ribosome-sequestering proteins and the ppGpp levels. a, The number of mRNA (black circles) and protein (blue circles) as a percentage of the total number of mRNA and protein, respectively, for the ribosome remodeling factor Rmf obtained during steady state growth are plotted against the growth rate for a number of cultures under varying degrees of carbon limitation. Data for mRNA abundance is obtained from Balakrishnan et $\mathrm{al}^{66}$ and for protein abundance is obtained from Mori et $\mathrm{al}^{65}$. b, Same as panel a, but for the mRNA and protein of the ribosome associated inhibitor gene raiA ${ }^{67}$. c, Same as panel a, but for the mRNA and protein of the gene encoding the hibernation promoting factor $h p f^{36}$. For each case shown in panels a-c, the protein and mRNA abundances approximately match each other within 2-fold, as is typical of many genes expressed during exponential growth. For Rmf, the protein level showed zeero for a number of conditions due to lack of detected peptides. d-f, The fractional number abundances of the mRNA (black circles) of $r m f$, raiA and $h p f$ are plotted against the relative ppGpp of the corresponding conditions. The black dash lines show the linear fit of the data. To directly compare the expression of $r m f$, raiA and $h p f$ with ppGpp levels, we fitted the mRNA vs GR data in panel a-c with $3^{\text {rd }}$-degree polynomials and estimated their mRNA levels at the same growth rates for which ppGpp was measured. The horizonal error bars in panels $\mathrm{d}-\mathrm{f}$ comes from ppGpp measurements, while the vertical error bars come from the polynomial fitting (95\% prediction interval). 
a

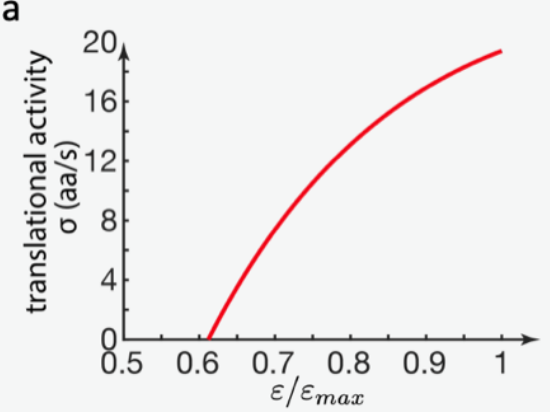

b

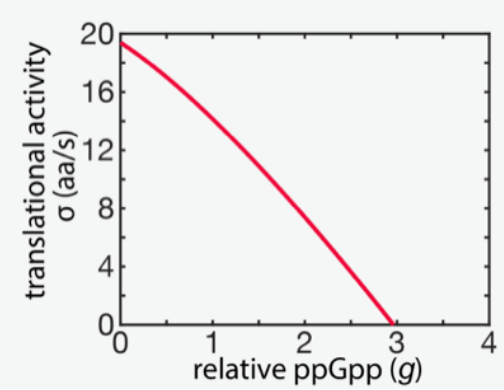

C

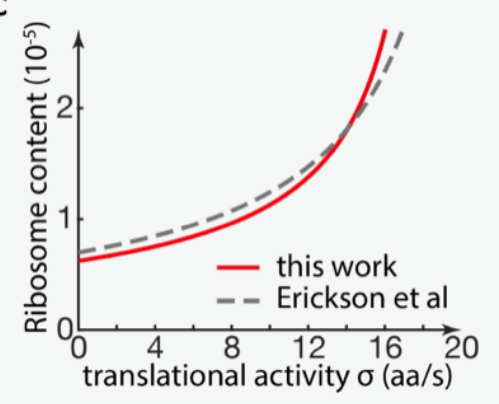

Extended Data Figure 4. a, Translational activity $\sigma$, defined as $\lambda / R$, and thus given by $\varepsilon \cdot(R-$ $H) / R$, is plotted against normalized elongation rate for the best-fit model parameters used in Fig. 3 . b, Translational activity $\sigma$ plotted against the relative ppGpp level $g$. The one-to-one relationship between the translational activity and the ppGpp level justifies the use of the translational activity as the dynamic variable in the kinetic model of diauxic transition developed by Erickson et $\mathrm{al}^{41}$. c, The plot of ribosomal content vs $\sigma$ used by Erickson et $\mathrm{al}^{41}$ as the regulatory function for ribosome biogenesis is shown as the grey dashed line. The same relation according to the model in Fig. 3 is shown as the solid red line. The two lines are very similar, indicating that Erickson et $\mathrm{al}^{41}$ correctly inferred the regulatory function even though the relation between $\sigma$ and $g$ (panel c) was not known at the time. 


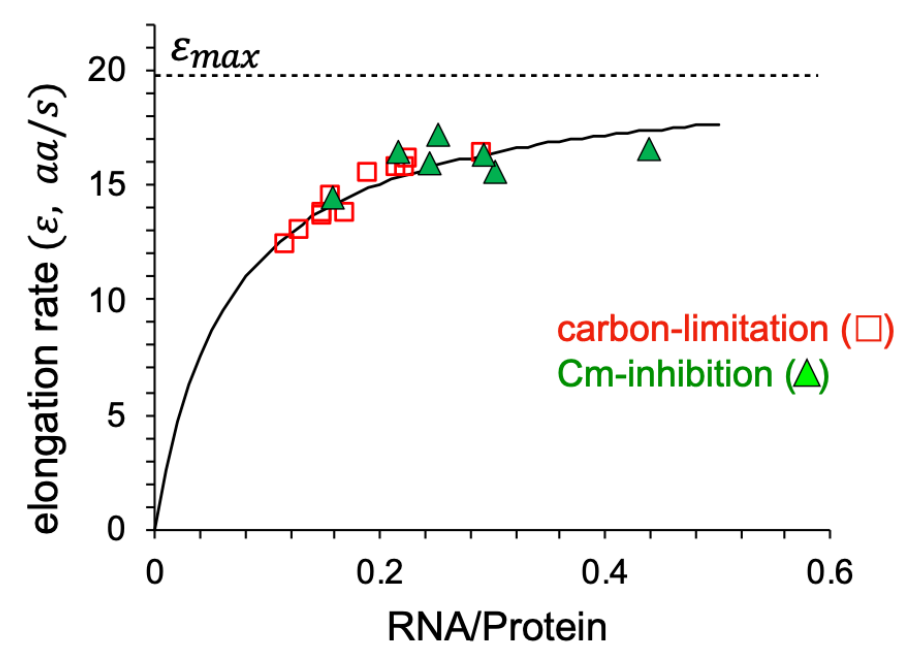

Extended Data Figure 5: Estimating the maximum translation elongation rate in steady-state growth. Translation elongation rates measured in the carbon-limited and chloramphenicol-treated cultures are plotted against the ratio of RNA and protein measured in the same conditions. The data are collectively fitted to the Michaelis-Menten relation $\varepsilon=\varepsilon_{\max } \cdot r /\left(K_{m}+r\right)$ where $r$ is the RNA-protein ratio. The best-fit gives $\varepsilon_{\max }=20.0 \pm 1.9 \mathrm{aa} / \mathrm{s}$ and $k_{m}=0.066$. The maximum elongation rate $\varepsilon_{\max }$ estimated this way is indistinguishable from that estimated from the relation between ppGpp and the elongation rate during growth transition (see Extended Data Fig. 1e). 
a

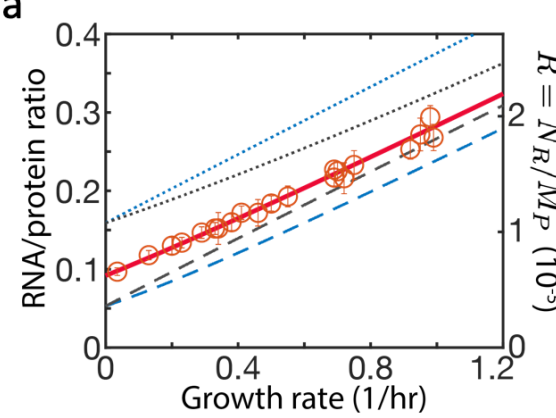

C

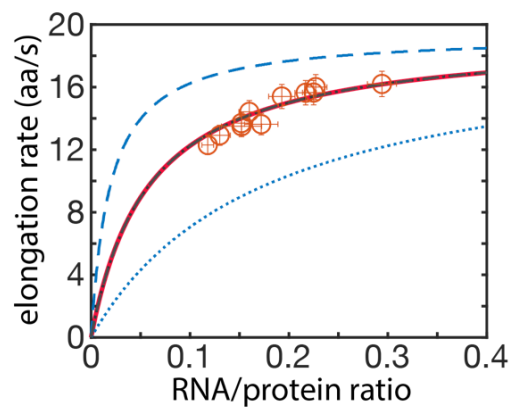

b

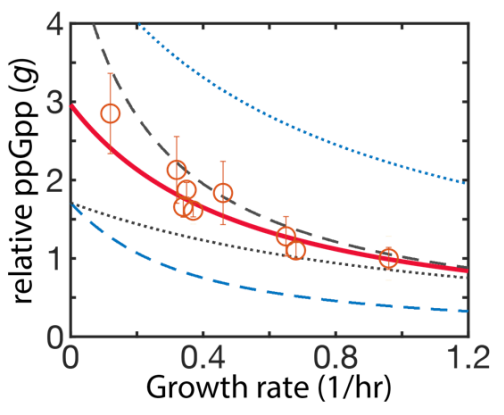

d

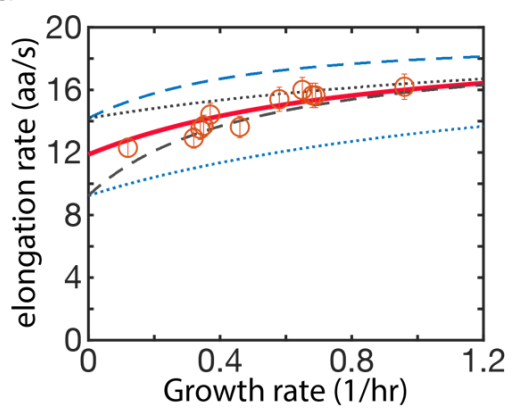

\begin{tabular}{|c|c|c|}
\hline & $a$ & $b$ \\
\hline- & $a^{*}$ & $b^{*}$ \\
\hline$\cdots \cdots \cdots$ & $3 a^{*}$ & $b^{*}$ \\
\hline--- & $a^{*} / 3$ & $b^{*}$ \\
\hline$\ldots \cdots \cdots$ & $a^{*}$ & $3 b^{*}$ \\
\hline--- & $a^{*}$ & $b^{*} / 3$ \\
\hline
\end{tabular}

Extended Data Figure 6: The effect of parameter choices on model output. The measured data points (red open circles) and the best-fit model outputs (solid red lines) in panels a-d are the same as those shown in Fig. 3b-e. When keeping parameter $a$ the same as the best-fit value $a^{*}$ while varying $b$ to be 3 -fold larger or smaller, the model gave predictions shown as the dotted and dash black lines, respectively. When keeping parameter $b$ the same as the best-fit value $b^{*}$ and varying $a$ to be 3-fold larger or smaller, the model gave predictions shown as the dotted and dash blue lines, respectively; See the legends table. The results show that model outputs are comparatively more sensitive to the choice of the parameter $a$ than $b$. Fortunately, the parameter $a$ involved in the relation between ppGpp and the ribosome content (see Eq. (3)) is well established by Fig. 3a. 

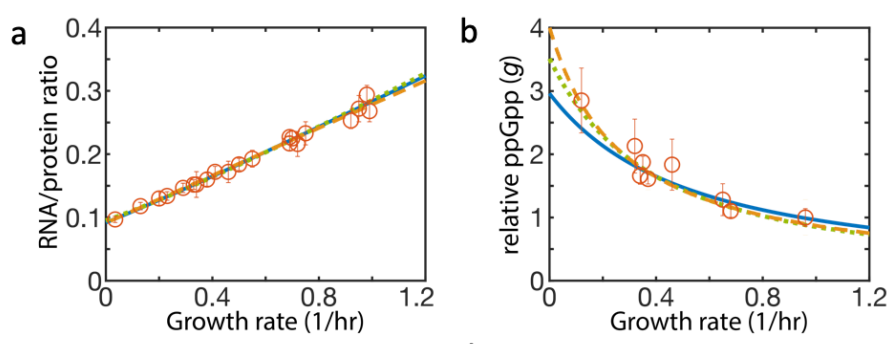

C

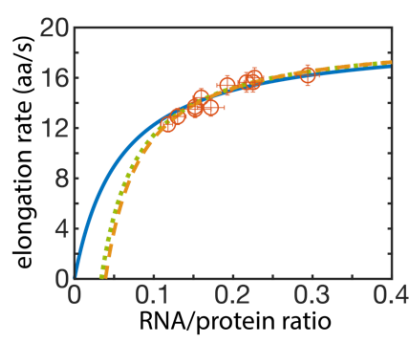

d
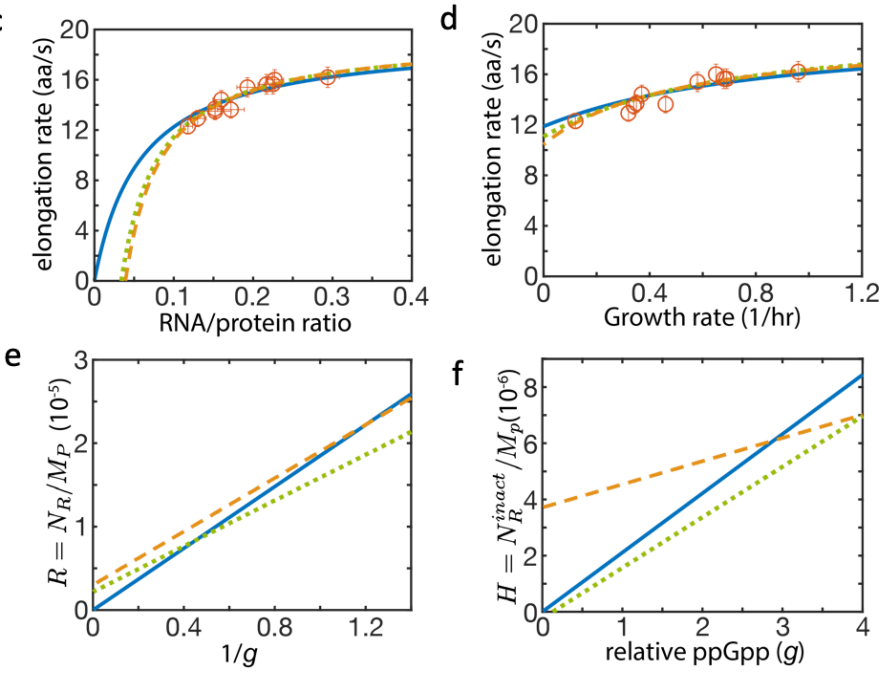

\begin{tabular}{|c|c|c|c|}
\hline & $\begin{array}{c}g_{H}=0 \\
g_{R}=\infty \\
\frac{r R N A}{R N A} \text { fitted }\end{array}$ & $\begin{array}{c}g_{H} \text { fitted } \\
g_{R} \text { fitted } \\
\frac{r R N A}{R N A}=0.86\end{array}$ & $\begin{array}{c}g_{H} \text { fitted } \\
g_{R} \text { fitted } \\
\frac{r R N A}{R N A} \text { fitted }\end{array}$ \\
\hline & & 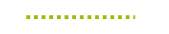 & ---- . \\
\hline$a$ & $1.85 \times 10^{-5}$ & $1.37 \times 10^{-5}$ & $1.6 \times 10^{-5}$ \\
\hline$b$ & $2.11 \times 10^{-6}$ & $1.8 \times 10^{-6}$ & $8.25 \times 10^{-7}$ \\
\hline$g_{R}$ & & 6.3 & 5.3 \\
\hline$g_{H}$ & & -0.13 & 4.5 \\
\hline$\frac{r R N A}{R N A}$ & 0.92 & & 1.03 \\
\hline \multicolumn{3}{|c|}{$R(g)=a \cdot\left(1 / g+1 / g_{R}\right)$} & $---(E 3)$ \\
\hline \multicolumn{3}{|c|}{$H(g)=b \cdot\left(g+g_{H}\right)$} & $---(E 4)$ \\
\hline
\end{tabular}

Extended Data Figure 7. The effect of constant offsets in the regulatory functions on model output. Here we investigate the effect of altered forms of regulatory functions from the simplest forms assumed in Eqs. (3) and (4). The altered forms are shown as Eqs. (E3) and (E4) below the legend table on the right, with constant offsets parameterized by $g_{R}$ and $g_{H}$. a-d show the same four outputs as in Extended Data Figure 6 with different modeling results. The table on the right compares the best-fit parameter values under different model settings. The solid blue line is the same as that shown in Fig. 3 (where offsets in $R(g)$ or $H(g)$ are not considered, left column of the legend). The orange dash lines are the best-fit of the model with $g_{R}$ and $g_{H}$ treated as fitting parameters also. Results of the fits are listed in the right column of the legend table. While the fits may be marginally better, one aspect of the fitting parameter becomes quite unreasonable: the relation between the ribosome content $\left(N_{R} / M_{P}\right)$ and the RNA-protein ratio is determined by the fraction of ribosome RNA within all RNA (the last row in legend table). In the case where all parameters are fitted (right column), the rRNA-RNA ratio is $\sim 1$, which is impossible given that tRNA comprises $\sim 10 \%$ of the total RNA. In comparison, the simplest model not considering offsets gave an rRNA-RNA ratio of $92 \%$ (left column) which is closer to the accepted range. If we fix the rRNA-RNA ratio to $86 \%$, estimated previously for a few growth conditions ${ }^{68,69}$, the bestfit model outputs are shown as the green dotted lines, with the best-fit parameters shown in the middle column. For the ease of assessing the effect of these offsets on the form of the regulatory functions $R(g)$ and $H(g)$, we display these forms for the 3 cases considered in panel $\mathbf{e}$ and $\mathbf{f}$, using the same line styles as those indicated in the legend table. We see that little difference is made to the model outputs despite substantial changes to the forms of the regulatory functions, thus indicating that these results are robust to the exact forms of the regulatory functions assumed. 


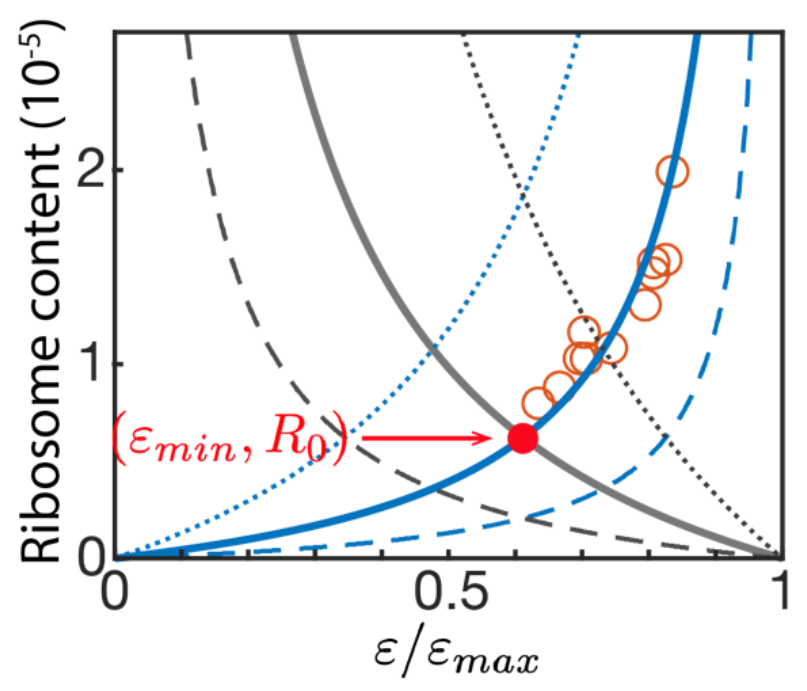

Extended Data Figure 8. Ribosome content are plotted against normalized elongation rate for various model parameters. Using the best-fit parameters in Fig. 3, total ribosome content (solid blue line) increases with elongation rate while inactive ribosome content (solid grey line) decrease with elongation rate. Their intersection (marked by the filled red circle) represents the elongation rate and ribosome content while growth rate approaches zero, i.e., $\varepsilon_{\min }$ and $R_{0}$, respectively. When the parameter $a$ is changed to 3-fold smaller or larger, the relation between total ribosome content and elongation rate is changed to the blue dashed or dotted lines, respectively. When the parameter $b$ is changed 3 -fold smaller or larger, the relation between total ribosome content and elongation rate is changed to the black dashed and dotted lines, respectively. As shown in the figure, the different parameters result in different values of the intersection point and hence different values of $\left(\varepsilon_{\text {min }}, R_{0}\right)$. 


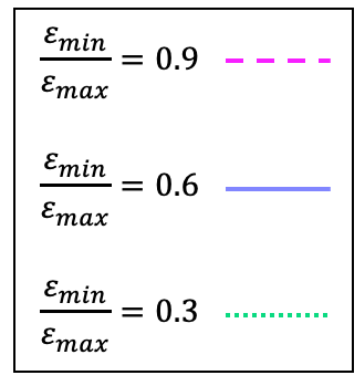

a

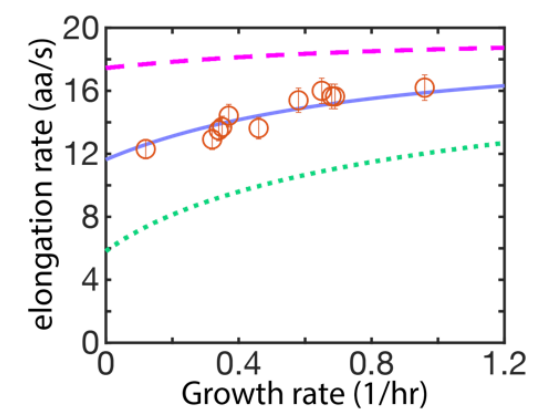

C

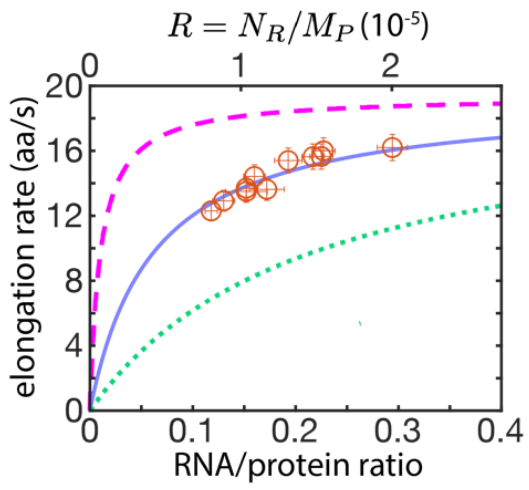

b

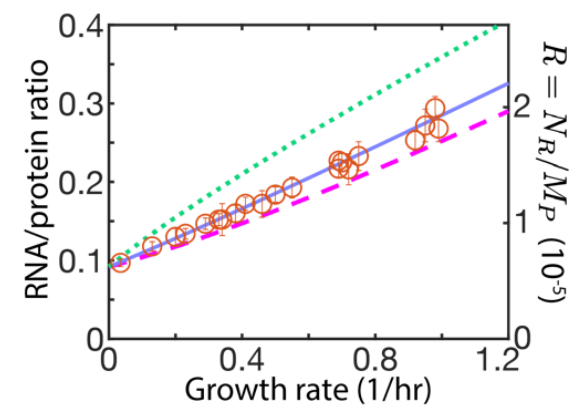

d

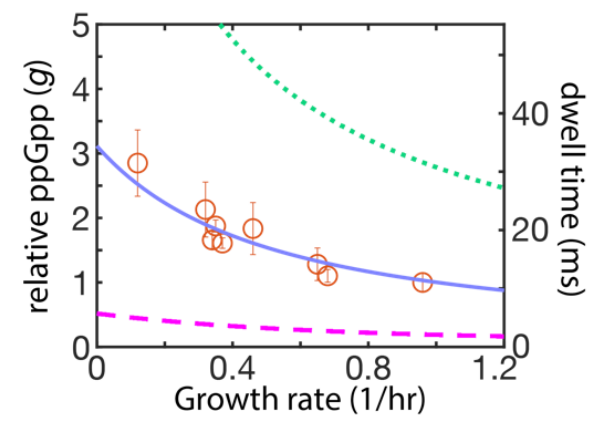

Extended Data Figure 9. The effect of the value of $\boldsymbol{\varepsilon}_{\min }$ on model output. As shown in Supplementary Note, the model defined by Eqs. (1) and (5) has a particularly simple solution for $\varepsilon_{\min }=\varepsilon_{\max } / 2$, where $\varepsilon_{\min }$ is the elongation rate as the growth rate $\lambda \rightarrow 0$. The best-fit output of the model has $\varepsilon_{\min }: \varepsilon_{\max } \approx 0.6$, which is close to the simple linear solution, thus rationalizing the approximate linear correlations observed in Fig. 3. In this figure, we show the model output for choices of the parameters $a$ and $b$ such that $\varepsilon_{\min }: \varepsilon_{\max }=0.3$ and 0.9 (dotted and dashed lines, respectively). The red open circles are the same data as those shown in Fig. 3. Deviations from the linear growth law are clearly seen for the expected RNA/protein ratio in panel b, with the high ratio of $\varepsilon_{\min }: \varepsilon_{\max }$ (dashed purple lines) exhibiting reduced ribosome content. In principle, large ER and reduced RNA/protein ratio are advantageous. However, this would lead to very low dwell time for ribosome on A-site as seen in panel d (right vertical axis): 10-30 ms for the best-fit parameters, and $<5 \mathrm{~ms}$ for the purple dashed line with ER maintained above $90 \%$ of $\varepsilon_{\max }$. 
a

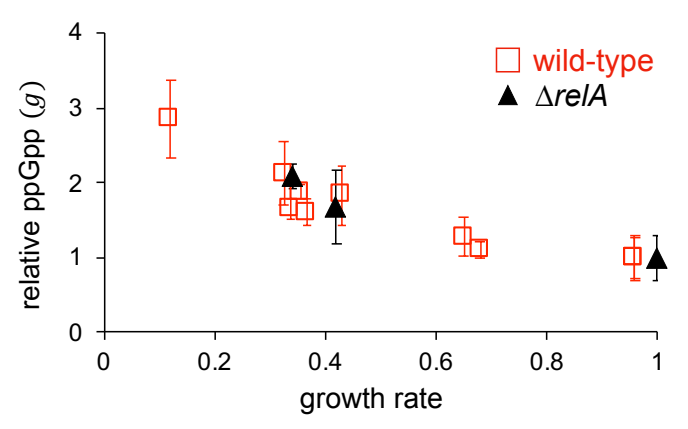

b

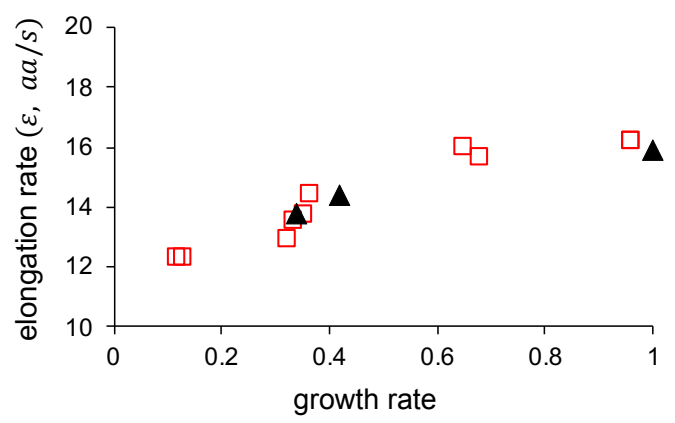

C

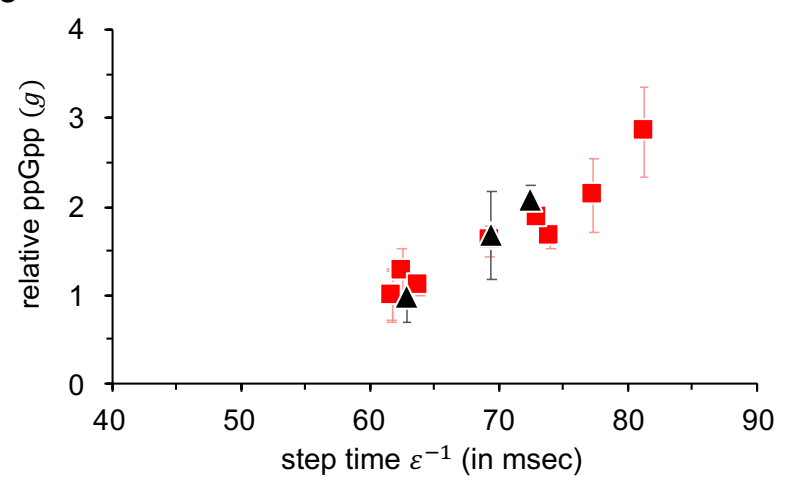

Extended Data Figure 10. Relation between the ppGpp level and elongation rate for the $\Delta$ relA strain in steady-state growth. a, ppGpp levels relative to that in the reference growth condition (wild type grown in minimal glucose medium) are plotted against the steady state growth rates for wild-type (red) and $\Delta$ relA (black) grown in various carbon sources (See Supplementary Table 1). Error bar represents the uncertainty in the linear fit over four measurements; see Supplementary Fig. 1. b, Translation elongation rates are plotted against the steady state growth rates for wildtype (red) and $\triangle$ relA (black) grown in various carbon sources (See Supplementary Table 1). c, Steady-state relative ppGpp levels are plotted against the reciprocal of the translational elongation rate for wild-type (red) and $\Delta$ relA (black) grown in the same conditions. Both the ppGpp levels and the elongation rates in steady state growth are not affected by relA deletion, unlike the case during diauxic transition shown in Extended Data Fig. 2. 


\section{References}

1. Neidhardt, F. C. GUEST COMMENTARY Bacterial Growth: Constant Obsession with dN/dt. J. Bacteriol. 181, 7405-7408 (1999).

2. Hui, S. et al. Quantitative proteomic analysis reveals a simple strategy of global resource allocation in bacteria. Mol Syst Biol 11, 784 (2015).

3. Si, F. et al. Invariance of Initiation Mass and Predictability of Cell Size in Escherichia coli. Curr. Biol. 27, 1278-1287 (2017).

4. Belliveau, N. M. et al. Fundamental limits on the rate of bacterial growth. bioRxiv (2020) doi:10.1101/2020.10.18.344382.

5. Baracchini, E. \& Bremer, H. Control of rRNA synthesis in Escherichia coli at increased rrn gene dosage: Role of guanosine tetraphosphate and ribosome feedback. J. Biol. Chem. 266, 11753-11760 (1991).

6. Paul, B. J., Ross, W., Gaal, T. \& Gourse, R. L. rRNA transcription in Escherichia coli. Annu. Rev. Genet. 38, 749-770 (2004).

7. Srivatsan, A. \& Wang, J. D. Control of bacterial transcription, translation and replication by (p)ppGpp. Curr. Opin. Microbiol. 11, 100-5 (2008).

8. Hauryliuk, V., Atkinson, G. C., Murakami, K. S., Tenson, T. \& Gerdes, K. Recent functional insights into the role of (p)ppGpp in bacterial physiology. Nat. Rev. Microbiol. 13, 298-309 (2015).

9. Irving, S. E., Choudhury, N. R. \& Corrigan, R. M. The stringent response and physiological roles of (pp)pGpp in bacteria. Nat. Rev. Microbiol. 19, 256-271 (2021).

10. Schaechter, M., MaalOe, O. \& Kjeldgaard, N. O. Dependency on Medium and Temperature of Cell Size and Chemical Composition during Balanced Growth of Salmonella typhimurium. J. Gen. Microbiol. 19, 592-606 (1958).

11. Maaløe, O. Regulation of the Protein-Synthesizing Machinery---Ribosomes, tRNA, Factors, and So On. in Biological Regulation and Development: Gene Expression (ed. Goldberger, R. F.) 487-542 (Springer US, 1979). doi:10.1007/978-1-4684-3417-0_12.

12. Scott, M., Gunderson, C. W., Mateescu, E. M., Zhang, Z. \& Hwa, T. Interdependence of Cell Growth. Science (80-. ). 330, 1099-1102 (2010).

13. You, C. et al. Coordination of bacterial proteome with metabolism by cyclic AMP signalling. (2013) doi:10.1038/nature12446.

14. Cashel, M. \& Gallant, J. Two compounds implicated in the function of RC gene. Nature 221, 838-841 (1969). 
15. Magnusson, L. U., Farewell, A. \& Nyström, T. ppGpp: A global regulator in Escherichia coli. Trends Microbiol. 13, 236-242 (2005).

16. Potrykus, K. \& Cashel, M. (p)ppGpp: Still magical? Annu. Rev. Microbiol. 62, 35-51 (2008).

17. Ronneau, S. \& Hallez, R. Make and break the alarmone: Regulation of (p)ppGpp synthetase/hydrolase enzymes in bacteria. FEMS Microbiol. Rev. 43, 389-400 (2019).

18. Bremer, H. \& Ehrenberg, M. Guanosine tetraphosphate as a global regulator of bacterial RNA synthesis: a model involving RNA polymerase pausing and queuing. $B B A-G e n e$ Struct. Expr. 1262, 15-36 (1995).

19. Traxler, M. F. et al. The global, ppGpp-mediated stringent response to amino acid starvation in Escherichia coli. Mol. Microbiol. 68, 1128-1148 (2008).

20. Lemke, J. J. et al. Direct regulation of Escherichia coli ribosomal protein promoters by the transcription factors ppGpp and DksA. Proc. Natl. Acad. Sci. 108, 5712-5717 (2011).

21. Sanchez-Vazquez, P., Dewey, C. N., Kitten, N., Ross, W. \& Gourse, R. L. Genome-wide effects on Escherichia coli transcription from ppGpp binding to its two sites on RNA polymerase. Proc. Natl. Acad. Sci. 116, 8310-8319 (2019).

22. Baracchini, E. \& Bremer, H. Stringent and growth control of rRNA synthesis in Escherichia coli are both mediated by ppGpp. J. Biol. Chem. 263, 2597-2602 (1988).

23. Harshman, R. B. \& Yamazaki, H. Formation of ppGpp in a Relaxed and Stringent Strain of Escherichia coli during Diauxie Lag. Biochemistry 10, 3980-3982 (1971).

24. Okano, H., Hermsen, R., Kochanowski, K. \& Hwa, T. Regulation underlying hierarchical and simultaneous utilization of carbon substrates by flux sensors in Escherichia coli. Nat. Microbiol. 5, 206-215 (2020).

25. Dai, X. et al. Reduction of translating ribosomes enables Escherichia coli to mantain elongation rates during slow growth. Nat. Microbiol. 2, 16231 (2016).

26. Dai, X. et al. Slowdown of translational elongation in Escherichia coli under hyperosmotic stress. MBio 9, (2018).

27. Volkov, I. L. et al. TRNA tracking for direct measurements of protein synthesis kinetics in live cells. Nat. Chem. Biol. 14, 618-626 (2018).

28. Volkov, I. L., Seefeldt, A. C. \& Johansson, M. Tracking of single tRNAs for translation kinetics measurements in chloramphenicol treated bacteria. Methods 162-163, 23-30 (2019).

29. Loveland, A. B. et al. Ribosome.RelA structures reveal the mechanism of stringent response activation. Elife 5, 1-23 (2016). 
30. Arenz, S. et al. The stringent factor RelA adopts an open conformation on the ribosome to stimulate ppGpp synthesis. Nucleic Acids Res. 44, 6471-6481 (2016).

31. Ryals, J., Little, R. \& Bremer, H. Control of rRNA and tRNA syntheses in Escherichia coli by guanosine tetraphosphate. J. Bacteriol. 151, 1261-1268 (1982).

32. Goldberg, A. L. \& St John, A. C. Intracellular protein degradation in mammalian and bacterial cells: Part 2. Annu. Rev. Biochem. 45, 747-803 (1976).

33. Nath, K. \& Koch, A. L. Protein degradation in Escherichia coli. II. Strain differences in the degradation of protein and nucleic acid resulting from starvation. J. Biol. Chem. 246, 6956-6967 (1971).

34. Mori, M., Schink, S., Erickson, D. W., Gerland, U. \& Hwa, T. Quantifying the benefit of a proteome reserve in fluctuating environments. Nat. Commun. 8, 1-8 (2017).

35. Izutsu, K., Wada, A. \& Wada, C. Expression of ribosome modulation factor (RMF) in Escherichia coli requires ppGpp. Genes to Cells 6, 665-676 (2001).

36. Ueta, M. et al. Role of HPF (hibernation promoting factor) in translational activity in Escherichia coli. J. Biochem. 143, 425-433 (2008).

37. Polikanov, Y. S., Blaha, G. M. \& Steitz, T. A. How hibernation factors RMF, HPF, and YfiA turn off protein synthesis. Science (80-. ). 336, 915-918 (2012).

38. Sarubbi, E., Rudd, K. E. \& Cashel, M. Basal ppGpp level adjustment shown by new spoT mutants affect steady state growth rates and rrnA ribosomal promoter regulation in Escherichia coli. MGG Mol. Gen. Genet. 213, 214-222 (1988).

39. Potrykus, K., Murphy, H., Philippe, N. \& Cashel, M. ppGpp is the major source of growth rate control in E. coli. Environ. Microbiol. 13, 563-575 (2011).

40. Gourse, R. L., Gaal, T., Bartlett, M. S., Appleman, J. A. \& Ross, W. rRNA Transcription and growth rate-dependent regulation of ribosome synthesis in Escherichia coli. Annu. Rev. Microbiol. 50, 645-677 (1996).

41. Erickson, D. W. et al. A global resource allocation strategy governs growth transition kinetics of Escherichia coli. Nature 551, 119-123 (2017).

42. Kjeldgaard, N. O., MaalOe, O. \& Schaechter, M. The Transition Between Different Physiological States During Balanced Growth of Salmonella typhimurium. J. Gen. Microbiol. 19, 607-616 (1958).

43. Samatova, E., Daberger, J., Liutkute, M. \& Rodnina, M. V. Translational Control by Ribosome Pausing in Bacteria: How a Non-uniform Pace of Translation Affects Protein Production and Folding. Front. Microbiol. 11, (2021).

44. Liu, Y. A code within the genetic code: Codon usage regulates co-translational protein 
folding. Cell Commun. Signal. 18, 1-9 (2020).

45. Mori, M., Marinari, E. \& De Martino, A. A yield-cost tradeoff governs Escherichia coli's decision between fermentation and respiration in carbon-limited growth. npj Syst. Biol. Appl. 5, (2019).

46. Gallant, J. A. Stringent Control in E. Coli. Annu. Rev. Genet. 13, 393-415 (1979).

47. Fernández-Coll, L. \& Cashel, M. Contributions of SpoT hydrolase, SpoT synthetase, and RelA synthetase to carbon source diauxic growth transitions in Escherichia coli. Front. Microbiol. 9, 1-13 (2018).

48. Jiang, M., Sullivan, S. M., Wout, P. K. \& Maddock, J. R. G-protein control of the ribosome-associated stress response protein SpoT. J. Bacteriol. 189, 6140-7 (2007).

49. Dennis, P. P. Regulation of ribosomal and transfer ribonucleic acid synthesis in Escherichia coli B-r. J. Biol. Chem. 247, 2842-2845 (1972).

50. Richter, D. Uncharged tRNA inhibits guanosine 3',5'-bis (diphosphate) 3'pyrophosphohydrolase [ppGppase], the spoT gene product, from Escherichia coli. $M G G$ Mol. Gen. Genet. 178, 325-327 (1980).

51. Li, S. H. J. et al. Escherichia coli translation strategies differ across carbon, nitrogen and phosphorus limitation conditions. Nat. Microbiol. 3, 939-947 (2018).

52. Hughes, J. \& Mellows, G. Inhibition of isoleucyl-transfer ribonucleic acid synthetase in Escherichia coli by pseudomonic acid. Biochem. J. 176, 305-318 (1978).

53. Bennett, P. M. \& Maaløe, O. The effects of fusidic acid on growth, ribosome synthesis and RNA metabolism in Escherichia coli. J. Mol. Biol. 90, 541-561 (1974).

54. Vadia, S. et al. Fatty Acid Availability Sets Cell Envelope Capacity and Dictates Microbial Cell Size. Curr. Biol. 27, 1757-1767.e5 (2017).

55. Hwa, T. Cell Behavior and Control. in The Physics of Living Matter: Space, Time and Information 85-134 (WORLD SCIENTIFIC, 2020). doi:10.1142/9789813239258_0003.

56. Cayley, S., Lewis, B. A., Guttman, H. J. \& Record, M. T. Characterization of the cytoplasm of Escherichia coli K-12 as a function of external osmolarity. Implications for protein-DNA interactions in vivo. J. Mol. Biol. 222, 281-300 (1991).

57. Soupene, E. et al. Physiological Studies of Escherichia coli Strain MG1655: Growth Defects and Apparent Cross-Regulation of Gene Expression. J. Bacteriol. 185, 5611-5626 (2003).

58. Brown, S. D. \& Jun, S. Complete Genome Sequence of Escherichia coli NCM3722. Genome Announc. 3, e00879-15 (2015). 
59. Datsenko, K. A. \& Wanner, B. L. One-step inactivation of chromosomal genes in Escherichia coli K-12 using PCR products. Proc. Natl. Acad. Sci. U. S. A. 97, 6640-5 (2000).

60. Schleif, R., Hess, W., Finkelstein, S. \& Ellis, D. Induction kinetics of the L arabinose operon of Escherichia coli. J. Bacteriol. 115, 9-14 (1973).

61. Cashel, M. The control of ribonucleic acid synthesis in Escherichia coli. IV. Relevance of unusual phosphorylated compounds from amino acid-starved stringent strains. J. Biol.

Chem. 244, 3133-3141 (1969).

62. Benthin, S., Nielsen, J. \& Villadsen, J. A simple and reliable method for the determination of cellular RNA content. Biotechnol. Tech. 5, 39-42 (1991).

63. Herbert, D., Phipps, P. J. \& Strange, R. E. Chemical Analysis of Microbial Cells. Methods Microbiol. 5, 209-344 (1971).

64. Brown, A., Fernández, I. S., Gordiyenko, Y. \& Ramakrishnan, V. Ribosome-dependent activation of stringent control. Nature 534, 277-280 (2016).

65. Mori, M. et al. From coarse to fine : The absolute Escherichia coli proteome under diverse growth conditions. Mol. Syst. Biol. 17, e9536 (2021).

66. Balakrishnan, R. et al. Principles of gene regulation quantitatively connect DNA to RNA and proteins in bacteria. bioRxiv 2021.05.24.445329 (2021)

doi:10.1101/2021.05.24.445329.

67. Agafonov, D. E., Kolb, V. A. \& Spirin, A. S. Ribosome-associated protein that inhibits translation at the aminoacyl-tRNA binding stage. EMBO Rep. 2, 399-402 (2001).

68. Bremer, H. \& Dennis, P. P. Modulation of Chemical Composition and Other Parameters of the Cell at Different Exponential Growth Rates. EcoSal Plus 3, (2008).

69. Harvey, R. J. \& Koch, A. L. How partially inhibitory concentrations of chloramphenicol affect the growth of Escherichia coli. Antimicrob. Agents Chemother. 18, 323-337 (1980). 


\section{Supplementary Information}

\section{Supplementary Tables}

Supplementary Table 1: Strains and growth conditions used in this study.

\begin{tabular}{|c|c|c|c|}
\hline strain name & description & growth condition & $\begin{array}{c}\text { growth } \\
\text { rate }\left(h^{-1}\right) \\
\end{array}$ \\
\hline NCM3722 1,2 & $\begin{array}{l}\text { Wild type } \\
\text { E. coli } \mathrm{K}-12\end{array}$ & $\begin{array}{l}\text { MOPS }+20 \mathrm{mM} \text { glucose } \\
\text { MOPS }+20 \mathrm{mM} \text { succinate } \\
\text { MOPS }+40 \mathrm{mM} \text { glycerol } \\
\text { MOPS }+30 \mathrm{mM} \text { acetate } \\
\text { MOPS }+3 \mathrm{mM} \text { mannose } \\
\text { MOPS }+20 \mathrm{mM} \text { aspartate }+10 \mathrm{mM} \mathrm{NH}_{4} \mathrm{Cl} \\
\text { MOPS } 0.4 \% \text { glycerol+10mM arginine } \\
\text { MOPS }+20 \mathrm{mM} \text { glutamate }+10 \mathrm{mM} \mathrm{NH}_{4} \mathrm{Cl}\end{array}$ & $\begin{array}{l}0.96 \\
0.68 \\
0.65 \\
0.43 \\
0.33 \\
0.35 \\
0.32 \\
0.13\end{array}$ \\
\hline NQ12613 & $\Delta p t s G$ & $\begin{array}{l}\text { MOPS }+20 \mathrm{mM} \text { glucose } \\
\text { MOPS }+20 \mathrm{mM} \text { glucose }+2 \mu \mathrm{M} \mathrm{Cm} \\
\text { MOPS }+20 \mathrm{mM} \text { glucose }+3 \mu \mathrm{M} \mathrm{Cm}\end{array}$ & $\begin{array}{l}0.36 \\
0.16 \\
0.12\end{array}$ \\
\hline $\begin{array}{l}\text { HE838 } \\
\text { (this work) }\end{array}$ & ArelA & $\begin{array}{l}\text { MOPS } 20 \mathrm{mM} \text { glucose } \\
\text { MOPS }+4 \mathrm{mM} \text { mannose } \\
\text { MOPS }+20 \mathrm{mM} \text { aspartate }+10 \mathrm{mM} \mathrm{NH}_{4} \mathrm{Cl}\end{array}$ & $\begin{array}{l}0.99 \\
0.42 \\
0.34\end{array}$ \\
\hline \multicolumn{2}{|c|}{$\begin{array}{l}\text { NCM } 3722 \text { and HE838 under } \\
\text { diauxic transition }\end{array}$} & MOPS+2mM glucose+40mM glycerol & N.A. \\
\hline
\end{tabular}

Supplementary Table 2: Primers used in this study.

\begin{tabular}{l|l|l}
\hline name & sequence & use \\
\hline relA-P1 & $\begin{array}{l}\text { cgatttcggcaggtctggtcctaaaggagaggacgatggttg } \\
\text { cggtaagatgtgtaggctggagctgcttc }\end{array}$ & $\begin{array}{l}\text { Chromosomal deletion of } \\
\text { relA gene }\end{array}$ \\
\hline relA-P2 & $\begin{array}{l}\text { atatcaatctacattgtagatacgagcaaatttcggcctaactccc } \\
\text { gtgcaattccggggatccgtcgacctg }\end{array}$ & $\begin{array}{l}\text { Chromosomal deletion of } \\
\text { relA gene }\end{array}$ \\
\hline relA-ver-R & tacgctactgtggatcataacctttc & $\begin{array}{l}\text { Verification of deletion of } \\
\text { relA gene }\end{array}$ \\
\hline
\end{tabular}

\footnotetext{
${ }^{1}$ Soupene, E. et al. Physiological Studies of Escherichia coli Strain MG1655: Growth Defects and Apparent CrossRegulation of Gene Expression. J. Bacteriol. 185, 5611-5626 (2003).

${ }^{2}$ Brown, S. D. \& Jun, S. Complete Genome Sequence of Escherichia coli NCM3722. Genome Announc. 3, e0087915 (2015).

${ }^{3}$ Dai, X. et al. Reduction of translating ribosomes enables Escherichia coli to mantain elongation rates during slow growth. Nat. Microbiol. 2, 16231 (2016).
} 


\section{Supplementary Figure}

a

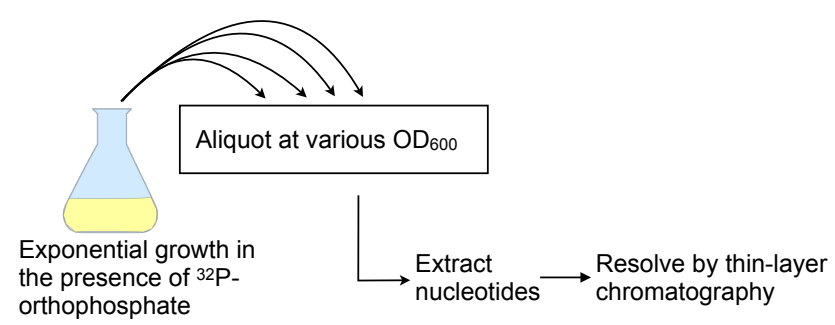

b

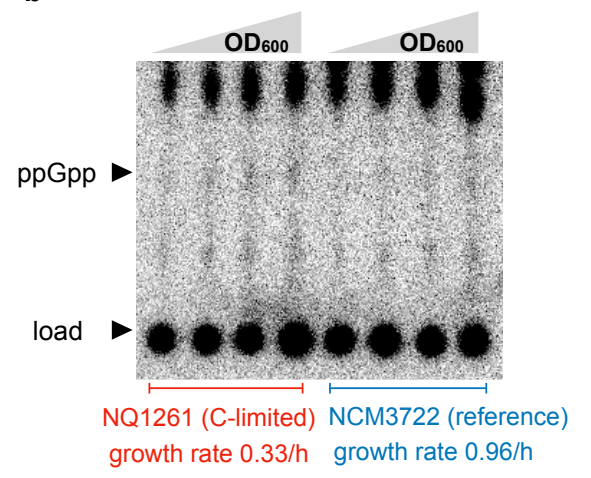

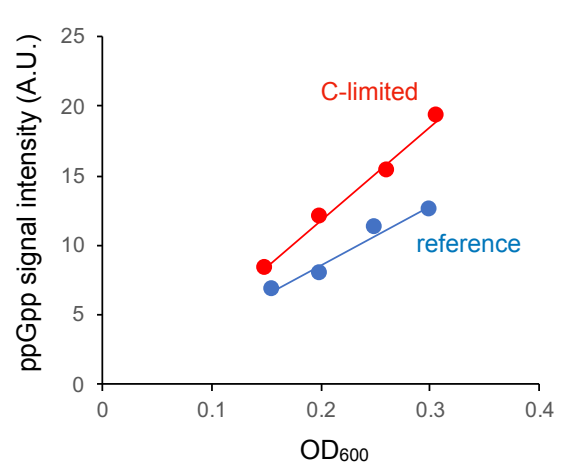

Supplementary Figure 1: Measurement of ppGpp levels in steady-state growth conditions. a, Scheme for ppGpp measurement under balanced growth. E. coli strains were grown in either glucose minimal media (reference condition, growth rate $=0.96 / \mathrm{h}$ ) or various poor carbons at different steady-state growth rates. At various $\mathrm{OD}_{600}$ values during the exponential growth phase of each culture, aliquots were withdrawn, nucleotides were extracted and ppGpp was resolved using TLC. b, An example of ppGpp measurement for the wild type strain (reference condition, blue) and NQ1261, a $\Delta p t s G$ strain defective in glucose uptake, both grown in glucose minimal medium (red). NQ1261 strain is used to create reduced growth on glucose (see Methods). Nucleotides extracted at four different $\mathrm{OD}_{600}$ were spotted on the bottom of TLC plates and ppGpp was resolved. c, ppGpp from the wild type and NQ1261 strains are plotted against OD 600 . The slope of each plot gives the ppGpp level (per $\mathrm{OD}_{600}$ ) for that strain and condition. In Fig. 2 of the main text, we report the ppGpp level relative to that in glucose steady state. In this case, the relative ppGpp level of NQ1261 strain grown in glucose is just the ratio of the slope of the red line to that of the blue line. Error bar in the estimate of ppGpp was taken to be the uncertainty in the slopes of the linear fit. 


\section{Supplementary Note}

\section{Dependence of the steady-state model on $\varepsilon_{\min }$ and $R_{0}$}

In the main text, Eq. (5) described the relationship between growth rate $\lambda$ and ppGpp level (or elongation rate via Eq. (1)) using parameter $a$ and $b$. Here we show that $a$ and $b$ can be expressed by $\varepsilon_{\min }$ and $R_{0}$, defined as the elongation rate (ER) and ribosomal abundance, respectively, when the growth rate approaches zero. Also, we show that when $\varepsilon_{\min }: \varepsilon_{\max }=1: 2$, the model yields simple dependences of $R$ and $g$ on $\lambda$.

In order for ER to be nonzero (with value $\varepsilon_{\min }$ ) when the growth rate approaches zero, we must have $R=H$ (with value $R_{0}$ ) according to Eq. (5). Using Eqs. (1) and (3), we can express $a$ in terms of $\varepsilon_{\min }$ and $R_{0}$ :

$$
a=c \cdot R_{0} \cdot(h-1),
$$

where we introduced $h \equiv \varepsilon_{\max } / \varepsilon_{\min }$ for convenience. Similarly, from Eqs. (1) and (4), the parameter $b$ can be expressed as

$$
b=\frac{R_{0}}{c \cdot(h-1)} .
$$

Conversely, we can express the two physiological parameters $R_{0}$ and $h$ in terms of the model parameters $a$ and $b$, with $R_{0}=\sqrt{a \cdot b}$ and $h=1+c \sqrt{a / b}$.

Substitute Eqs. (3), (S1) and (S2) into Eq. (5), we obtain a relationship between $\lambda$ and $R$ with $h$ and $R_{0}$ being the parameters:

$$
\lambda=\varepsilon_{\max } \frac{R^{2}-R_{0}^{2}}{R+R_{0} \cdot(h-1)} .
$$

Notice that when $h=2$ (or $\varepsilon_{\min }: \varepsilon_{\max }=1: 2$ ), Eq. (S3) is reduced to $\lambda=\varepsilon_{\max } \cdot\left(R-R_{0}\right)$, or

$$
R=R_{0}+\lambda / \varepsilon_{\max }
$$

i.e., an exact linear relationship between $R$ and $\lambda$ with $1 / \varepsilon_{\max }$ being the slope of $R-\lambda$ plot.

We can similarly work out the relation between $g$ and $\lambda$. From Eq. (S1) above, we can rewrite Eq. (3) of the main text as $R=R_{0} c \cdot(h-1) / g$. Defining the value of $g$ as the growth rate $\lambda \rightarrow 0$ as $g_{0}$, we have $c \cdot(h-1)=g_{0}$, or

$$
R=R_{0} \cdot g_{0} / g
$$


Substituting Eq. (S5) into Eq. (S3), we obtain

$$
\lambda=\varepsilon_{\max } R_{0} \frac{\left(g_{0} / g\right)^{2}-1}{g_{0} / g+(h-1)}
$$

For $h=2$ (or $\left.\varepsilon_{\min }: \varepsilon_{\max }=1: 2\right)$, Eq. (S6) is reduced to $\lambda=\varepsilon_{\max } R_{0} \cdot\left(g_{0} / g-1\right)$, or

$$
g=\frac{g_{0}}{1+\lambda /\left(\varepsilon_{\max } R_{0}\right)}
$$

i.e., a simple hyperbolic dependence of the ppGpp level on the growth rate $\lambda$. Alternatively, $g^{-1}$ has a simple linear dependence on $\lambda$.

Lastly, we examine the relation between $R$ and $\varepsilon$. Inverting Eq. (1) of the main text, we have

$$
\varepsilon=\frac{\varepsilon_{\max }}{1+g / c}
$$

Further using Eq. (S5) and $g_{0}=c \cdot(h-1)$, we obtain

$$
\varepsilon=\frac{\varepsilon_{\max }}{1+(h-1) R_{0} / R}
$$

which is generally of the Michaelis form, $\varepsilon_{\max }$ being the maximal ER. For $h=2$, the "Michaelis constant" becomes simply $R_{0}$. 